\title{
Heavy vector-like top partners at the LHC and flavour constraints
}

\author{
Giacomo Cacciapaglia, ${ }^{a, b}$ Aldo Deandrea, ${ }^{b}$ Luca Panizzi, ${ }^{b}$ Naveen Gaur, ${ }^{c}$ \\ Daisuke Harada $^{d}$ and Yasuhiro Okada ${ }^{d, e}$ \\ ${ }^{a}$ King's College London, Department of Physics, \\ Strand, London WC2R 2LS, U.K. \\ ${ }^{b}$ Université de Lyon, France; Université Lyon 1, \\ CNRS/IN2P3, UMR5822 IPNL, F-69622 Villeurbanne Cedex, France \\ ${ }^{c}$ Department of Physics, Dyal Singh College (University of Delhi), \\ Lodi Road, New Delhi - 110003, India \\ ${ }^{d}$ KEK Theory Center, Institute of Particle and Nuclear Studies, KEK, \\ 1-1 Oho, Tsukuba, Ibaraki 305-0801, Japan \\ e Department of Particle and Nuclear Physics, \\ Graduate University for Advanced Studies (Sokendai), \\ 1-1 Oho, Tsukuba, Ibaraki 305-0801, Japan \\ E-mail: cacciapaglia@ipnl.in2p3.fr, deandrea@ipnl.in2p3.fr, \\ panizzi@ipnl.in2p3.fr, gaur.nav@gmail.com, dharada@post.kek.jp, \\ yasuhiro.okada@kek.jp
}

ABSTRACT: We consider the phenomenology at the Large Hadron Collider of new heavy vector-like quarks which couple mainly to the third generation quarks via Yukawa interactions, with special emphasis on non-standard doublet representations which are less constrained from present data. We also discuss in detail the flavour limits at tree level and loop level and implications of a generalised CKM mixing matrix to these cases.

Keywords: Beyond Standard Model, B-Physics, Heavy Quark Physics 


\section{Contents}

1 Introduction $\quad 2$

2 The effective interactions 3

2.1 One flavour mixing 4

2.2 Three flavour mixing 4

2.3 Mixing matrices 6

2.4 Charged gauge boson: $W^{ \pm}$couplings and CKM matrix 7

2.5 Neutral gauge boson: $Z^{0}$ couplings and FCNCs 8

2.6 Higgs boson couplings 9

3 Tree level bounds $\quad 9$

$\begin{array}{llr}3.1 & \text { Precision electroweak measurements } & 9\end{array}$

$\begin{array}{lll}3.2 & \text { Direct bounds at the LHC (CMS) } & 10\end{array}$

$3.3 D_{0}-\bar{D}_{0}$ mixing and $D_{0} \rightarrow l^{+} l^{-}$decays 11

3.4 Rare decays of $D$ mesons 13

3.5 Top quark FCNC rare decays: $t \rightarrow Z c, Z u \quad 14$

$\begin{array}{lll}3.6 & \text { Atomic parity violation } & 15\end{array}$

$\begin{array}{lll}\text { 3.7 CKM matrix } & 16\end{array}$

4 Loop constraints and couplings $\quad 17$

$\begin{array}{lll}4.1 & \text { Kaon sector: } K^{0}-\bar{K}^{0} \text { mixing } & 18\end{array}$

$4.2 B$ meson sector: $B_{d}^{0}-\bar{B}_{d}^{0}$ mixing $\quad 20$

$4.3 B$ meson sector: $B_{s}^{0}-\bar{B}_{s}^{0}$ mixing 22

4.4 Coupling of the Higgs to gluons and photons 24

5 LHC phenomenology $\quad \mathbf{2 6}$

$\begin{array}{lll}5.1 \text { Decays } & 27\end{array}$

5.2 Single production 27

$\begin{array}{ll}\text { 5.2.1 Framework of event generation and analysis } & 29\end{array}$

5.2.2 Signature $p p \rightarrow t^{\prime}(\rightarrow t Z) j \rightarrow b j \ell^{ \pm} E_{T} \quad 30$

5.2.3 Signature $p p \rightarrow t^{\prime}(\rightarrow t Z) j \rightarrow b \ell^{ \pm} \ell^{\mp} \ell^{ \pm} j E_{T}$

5.2.4 Signature $p p \rightarrow t^{\prime}(\rightarrow t H) j \rightarrow b \bar{b} b j \ell^{ \pm} E_{T} \quad 35$

5.2.5 Signature $p p \rightarrow t^{\prime}(\rightarrow t Z) j \rightarrow b j j j \ell^{ \pm} E_{T} \quad 35$

5.2.6 Signature $p p \rightarrow t^{\prime}(\rightarrow j H) j \rightarrow j j \bar{b} b$, in the case $\sin \theta^{R}=0 . \quad 36$

6 Conclusions 38

$\begin{array}{ll}\text { A Expansion of the CKM matrix } & 39\end{array}$

B Notation for the meson mixing $\quad 40$ 


\section{Introduction}

Heavy vector-like fermions are not present in the Standard Model (SM), however they naturally arise near the electroweak scale in many extensions of new physics. Flavour hierarchies among SM masses for fermions and their mixings are most often generated through their dynamical mixing with vector-like fermions. These particles are indeed present in many models of new physics, like for example extra dimensional, Little Higgs and dynamical models. More recently the possibility of gauging the Standard Model flavour group requiring anomaly cancellation with the addition of new vector-like fermions was studied with explicit model constructions which show the possibility of a relatively low new gauge flavour bosons scale [1]. Moreover, in this case the vector-like quarks play an important role both for anomaly cancellation and for the mechanism of generation of fermion masses (see [2] for an example with left-right symmetry). The mixing of vector-like quarks with the other three generations and in particular the top quark is also a common feature in Little Higgs [3-6] and composite Higgs models [7] based on top condensation [8-12]. The Large Hadron Collider (LHC) is collecting data that allows to test and discover this sector quite soon. Typical cross-sections for pair and single production of heavy vector-like quarks are fairly large and within short term reach for the LHC experiments in the few hundred $\mathrm{GeV}$ mass range, as it will be discussed in detail in the following. Assuming some properties, the existing collider and precision data place limits on the new heavy quarks and set the lowest mass scale for these states. Direct searches, for example, give mass constraints in the $300 \mathrm{GeV}$ range, assuming a charged current decay chain [13]. In this work, we will focus on a particular example: namely, new coloured quarks that are doublets under the SM SU(2) symmetry, but with non-standard hypercharge. The resulting Yukawa couplings are less constrained by precision measurements than in other cases [14]. Moreover, a fermion with such quantum numbers is present in models of composite Higgs boson with extended custodial symmetry [20], engineered to protect the left-handed $Z b b$ coupling from large corrections. In this model, the gauge symmetry is extended to $\mathrm{SU}(2)_{L} \times \mathrm{SU}(2)_{R}$ in the bulk, and the left-handed top and bottom doublet comes in a bi-doublet of the two SU(2). The bi-doublet naturally contains a doublet with non-standard hypercharge. Moreover, the boundary conditions are such that the resonances from the non-standard doublet are parameterically lighter that the others [21], therefore one can approximate the phenomenology of such models by the addition of a massive doublet only, after integrating out the other more massive resonances.

The paper is organised as follows. In section 2 we describe the effective Yukawa interactions we consider for this study. We assume the presence of the SM quarks and of the standard Higgs doublet and couple them to the vector-like quarks. The CabibboKobayashi-Maskawa (CKM) flavour structure and the couplings to the standard gauge bosons and Higgs particle are discussed in detail. In section 3 we discuss the electroweak precision tests and the tree level bounds which define the accessible parameter space. In section 4 we explore the loop effects in the Kaon and B meson sector together with loop effects on the couplings of the Higgs boson to gluons and photons. In section 5 we study the LHC phenomenology for the production and the decay of vector-like quarks with a 
detailed simulation of various decay modes obtained from single production of a heavy vector-like top partner. Finally we give our conclusions. Two appendices contain more detailed material concerning the expansion of the CKM matrix and the notations used for the meson mixing formalism.

\section{The effective interactions}

We assume that the new fermions interact with the SM fermions via Yukawa interactions, therefore the quantum numbers of the new fermions with respect to the weak $\mathrm{SU}(2)_{L} \times$ $\mathrm{U}(1)_{Y}$ gauge group are limited by the requirement of an interaction with the Higgs doublet and one of the SM fermions. A complete survey of all the possibilities is given in [14] (see also $[15,16]$ for previous studies). Here we are interested mainly in the case of a new coloured fermion that transforms like a doublet of $\mathrm{SU}(2)$ with hypercharge $7 / 6$, because the bounds are milder in this case. We will therefore introduce the notation in this specific case. In the quark sector, a SM family contains a doublet $q_{L}=\left\{u_{L}, d_{L}\right\}^{T}=(2,1 / 6)$ and two singlets $u_{R}=(1,2 / 3)$ and $d_{R}=(1,-1 / 3)$, that couple to each other via the Higgs $H=(2,1 / 2)$. Here and in the following, $L$ and $R$ label respectively the left-handed or right-handed chirality of the field. The SM Yukawa couplings are:

$$
\mathcal{L}_{\text {Yukawa }}=-y_{u} \bar{q}_{L} H^{c} u_{R}-y_{d} \bar{q}_{L} H d_{R}+\text { h.c. } ;
$$

the up-type quarks (top), therefore, couple to the charge-conjugate of the Higgs boson. If we extend the SM with a new fermion $\psi=(2,7 / 6)=\{X, U\}^{T}$, it is possible to write down a Yukawa coupling between the new fermion and the up-type singlets via the Higgs boson $H$. The Yukawa sector that we will consider here is

$$
\begin{aligned}
\mathcal{L}_{\text {Yukawa }} & =-y_{u} \bar{q}_{L} H^{c} u_{R}-\lambda \bar{\psi}_{L} H u_{R}-M \bar{\psi}_{L} \psi_{R}+\text { h.c. } \\
& =-\frac{y_{u} v}{\sqrt{2}} \bar{u}_{L} u_{R}-\frac{\lambda v}{\sqrt{2}} \bar{U}_{L} u_{R}-M\left(\bar{U}_{L} U_{R}+\bar{X}_{L} X_{R}\right)+\text { h.c. }
\end{aligned}
$$

The new massive fermion contains a quark $X$ with electric charge $5 / 3$, and a fourth up quark which, however, has different gauge couplings with respect to the SM up quarks. The Higgs vacuum expectation value $(\mathrm{VEV}) v$ induces a mass mixing with the up quarks, therefore the mass eigenstates will be a mixture of the SM and new fermions. The new Yukawa couplings $\lambda$ connecting the heavy quarks with the SM ones will generate flavour mixing between the new states and the SM quarks in the up sector, while the down sector is left untouched. We will first review the simple limit of just one flavour mixing [14], in which the new state only mixes with the third generation: this is an interesting and physical limit because of the simpler notation and the fact that mixing with the light generations are much more tightly constrained. We will then give the more general parameterisation of the full three flavour mixing, which will show a very peculiar flavour structure, which is not flavour minimal and still can be consistent with present bounds with non-negligible couplings. 


\subsection{One flavour mixing}

In our previous paper [14], we found that the most general mass terms, including the Yukawa interactions, is

$$
\mathcal{L}_{\text {mass }}=-\tilde{m}_{t} \bar{u}_{L} u_{R}-x \bar{U}_{L} u_{R}-M \bar{U}_{L} U_{R}+\text { h.c. } .
$$

where $\tilde{m}_{t}=\frac{y_{u} v}{\sqrt{2}}$ and $x=\frac{\lambda v}{\sqrt{2}}$ can be chosen real thanks to a phase redefinition of $U$. It can be diagonalised by

$$
\left(\begin{array}{cc}
\cos \theta_{u}^{L} & -\sin \theta_{u}^{L} \\
\sin \theta_{u}^{L} & \cos \theta_{u}^{L}
\end{array}\right)\left(\begin{array}{cc}
\tilde{m}_{t} & 0 \\
x & M
\end{array}\right)\left(\begin{array}{cc}
\cos \theta_{u}^{R} & \sin \theta_{u}^{R} \\
-\sin \theta_{u}^{R} & \cos \theta_{u}^{R}
\end{array}\right)=\left(\begin{array}{cc}
m_{t} & 0 \\
0 & m_{t^{\prime}}
\end{array}\right)
$$

and the relations between parameters and masses and mixing angles are [14]

$$
\begin{aligned}
\tilde{m}_{t}^{2} & =\frac{y_{u}^{2} v^{2}}{2}=m_{t}^{2}\left(1+\frac{x^{2}}{M^{2}-m_{t}^{2}}\right), & m_{t^{\prime}}^{2} & =M^{2}\left(1+\frac{x^{2}}{M^{2}-m_{t}^{2}}\right), \\
\sin \theta_{u}^{R} & =\frac{M x}{\sqrt{\left(M^{2}-m_{t}^{2}\right)^{2}+M^{2} x^{2}}}, & \sin \theta_{u}^{L} & =\frac{m_{t}}{M} \sin \theta_{u}^{R} .
\end{aligned}
$$

We can however use those relations to express all parameters in terms of physical observables, like for example $m_{t}, m_{t^{\prime}}$ and $\theta_{u}^{R}$ :

$$
\begin{aligned}
M=m_{X} & =\sqrt{m_{t^{\prime}}^{2}+m_{t}^{2} \tan ^{2} \theta_{u}^{R}} \cos \theta_{u}^{R}, \\
\tilde{m}_{t} & =\frac{m_{t} m_{t^{\prime}}}{\sqrt{m_{t^{\prime}}^{2}+m_{t}^{2} \tan ^{2} \theta_{u}^{R}}} \frac{1}{\cos \theta_{u}^{R}}=\frac{m_{t} m_{t^{\prime}}}{m_{X}}, \\
x & =\frac{m_{t^{\prime}}^{2}-m_{t}^{2}}{\sqrt{m_{t^{\prime}}^{2}+m_{t}^{2} \tan ^{2} \theta_{u}^{R}}} \sin \theta_{u}^{R}=\frac{m_{t^{\prime}}^{2}-m_{t}^{2}}{m_{X}} \sin \theta_{u}^{R} \cos \theta_{u}^{R}, \\
\sin \theta_{u}^{L} & =\frac{m_{t}}{\sqrt{m_{t^{\prime}}^{2}+m_{t}^{2} \tan ^{2} \theta_{u}^{R}}} \tan \theta_{u}^{R}=\frac{m_{t}}{m_{X}} \sin \theta_{u}^{R} .
\end{aligned}
$$

In the rest of the paper we will use $m_{t^{\prime}}$ and $\theta_{u}^{R}$ as the two free parameters of the model, and the previous relations allows to calculate the fundamental parameters $x$ and $M$.

\subsection{Three flavour mixing}

If we consider the three families of quarks in the SM, the Yukawa couplings become matrices in flavour space, while $\lambda$ is a vector. With explicit flavour indices $i, j=1,2,3$, the Yukawa interactions are:

$$
\mathcal{L}_{\text {yuk }}=-y_{u}^{i, j} \bar{Q}_{L}^{i} H^{c} u_{R}^{j}-y_{d}^{i, j} \bar{Q}_{L}^{i} H d_{R}^{j}-\lambda^{j} \bar{\psi}_{L} H u_{R}^{j}
$$

In the following we will describe a convenient method to diagonalise the resulting mass terms. First, we can use the SM flavour symmetry to rewrite the standard Yukawa couplings as:

$$
y_{u}^{i, j}=\operatorname{diag}\left(y_{u}, y_{c}, y_{t}\right), \quad y_{d}^{i, j}=\tilde{V}_{C K M} \cdot \operatorname{diag}\left(y_{d}, y_{s}, y_{b}\right) .
$$


The matrix $\tilde{V}_{C K M}$ is the misalignment between left-handed ups and downs and it would correspond to the Cabibbo-Kobayashi-Maskawa matrix in the absence of the new fermion. The tilde signifies that this is not the case, as we will see. After the Higgs develops a VEV, $\langle H\rangle=v / \sqrt{2}$, the mass matrix for the fermions can be written (in this basis) as

$$
\begin{aligned}
& \mathcal{L}_{\text {mass }}=-\left(\bar{d}_{L}, \bar{s}_{L}, \bar{b}_{L}\right) \cdot \tilde{V}_{C K M} \cdot\left(\begin{array}{ccc}
\tilde{m}_{d} & & \\
& \tilde{m}_{s} & \\
& & \tilde{m}_{b}
\end{array}\right) \cdot\left(\begin{array}{c}
d_{R} \\
s_{R} \\
b_{R}
\end{array}\right)
\end{aligned}
$$

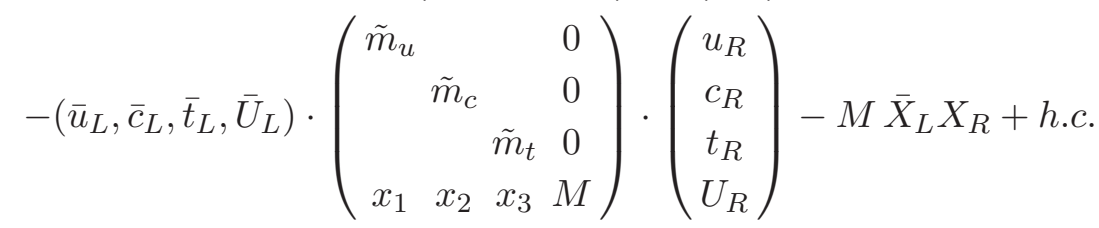

where $\tilde{m}_{x}=\frac{y_{x} v}{\sqrt{2}}$ and $x_{i}=\frac{\lambda^{i} v}{\sqrt{2}}$. For the down type quarks, the tilded masses are equal to the physical masses, as in the SM. For the up type quarks, there will be corrections coming from the mixing with the heavy $U$. Using the phase of $U$, we can show that $M$ and one of the $x$, say $x_{3}$, can be made real, while two physical phases are still present on $x_{1}$ and $x_{2}$. This will be important for $\mathrm{CP}$ violation. In the following we will take $x_{3}$ real, so that the formulas in the previous section can be used in this case without any change.

The mass matrix for the up quarks $M_{u}$ can be diagonalised by two unitary 4 by 4 matrices:

$$
M_{u}=V_{L} \cdot\left(\begin{array}{cccc}
m_{u} & & & \\
& m_{c} & & \\
& & m_{t} & \\
& & & m_{t^{\prime}}
\end{array}\right) \cdot V_{R}^{\dagger}
$$

$V_{L}$, that describes the mixing in the left-handed sector, is the unitary matrix that diagonalises the unitary matrix

$$
M_{u} \cdot M_{u}^{\dagger}=\left(\begin{array}{cccc}
\tilde{m}_{u}^{2} & 0 & 0 & x_{1}^{*} \tilde{m}_{u} \\
0 & \tilde{m}_{c}^{2} & 0 & x_{2}^{*} \tilde{m}_{c} \\
0 & 0 & \tilde{m}_{t}^{2} & x_{3} \tilde{m}_{t} \\
x_{1} \tilde{m}_{u} & x_{2} \tilde{m}_{c} & x_{3} \tilde{m}_{t} & M^{2}+\left|x_{1}\right|^{2}+\left|x_{2}\right|^{2}+x_{3}^{2}
\end{array}\right) .
$$

From this matrix we can see two things: a SM quark $q=u, c, t$ is exactly massless in the limit $\tilde{m}_{q} \rightarrow 0$, therefore the masses of the light quarks are always proportional to $\tilde{m}_{q}$; the mixing angles with the heavy $t^{\prime}$ are suppressed by $\tilde{m}_{q} / M \sim m_{q} / m_{t^{\prime}}$.

On the other hand, $V_{R}$, that describes the mixing in the right-handed sector, diagonalises

$$
M_{u}^{\dagger} \cdot M_{u}=\left(\begin{array}{cccc}
\tilde{m}_{u}^{2}+\left|x_{1}\right|^{2} & x_{1}^{*} x_{2} & x_{1}^{*} x_{3} & x_{1}^{*} M \\
x_{2}^{*} x_{1} & \tilde{m}_{c}^{2}+\left|x_{2}\right|^{2} & x_{2}^{*} x_{3} & x_{2}^{*} M \\
x_{3} x_{1} & x_{3} x_{2} & \tilde{m}_{t}^{2}+x_{3}^{2} & x_{3} M \\
x_{1} M & x_{2} M & x_{3} M & M^{2}
\end{array}\right)
$$


Here we see that the mixing is only suppressed by the $x$ 's couplings and that it is present also in the limit of massless SM quarks $\tilde{m}_{q} \rightarrow 0$. Therefore, it will be the couplings of the right-handed quarks to pose the most serious flavour constraints.

\subsection{Mixing matrices}

The two mixing matrices $V_{L}$ and $V_{R}$ can be calculated in an approximate way: we can assume that the parameters relative to the mass and mixing of the light two generations are small (therefore, $\tilde{m}_{u}, \tilde{m}_{c}, x_{1}$ and $x_{2}$ are of order $\epsilon$ compared to $\tilde{m}_{t}, M$ and $x_{3}$ ). We can then calculate the matrices in an expansion in $\epsilon$. A good trick is to first diagonalise the top-heavy fermion sector with the formulas in section 2.1 ; then, we can diagonalise the resulting matrix in the given expansion.

For the right-handed matrix, the result, up to order $\epsilon^{2}$, is:

$$
V_{R}^{i j}=\left(\begin{array}{ccc}
1-\frac{\left|x_{1}\right|^{2}}{2 m_{X}^{2}} & -\frac{x_{1}^{*} x_{2} m_{c}^{2}}{\left(m_{c}^{2}-m_{u}^{2}\right) m_{X}^{2}} & -\frac{x_{1}^{*} \sin \theta_{R}}{m_{X}} \\
\frac{x_{1} x_{2}^{*} m_{u}^{2}}{\left(m_{c}^{2}-m_{u}^{2}\right) m_{X}^{2}} & 1-\frac{\left|x_{2}\right|^{2}}{2 m_{X}^{2}} & -\frac{x_{2}^{*} \sin \theta_{R}}{m_{X}} \\
0 & 0 & \cos \theta_{R}+\frac{\left(m_{t^{\prime}}^{2}+m_{t}^{2}\right)\left(\left|x_{1}\right|^{2}+\left|x_{2}\right|^{2}\right) \cos \theta_{R} \sin ^{2} \theta_{R}}{2\left(m_{t^{\prime}}^{2}-m_{t}^{2}\right) m_{X}^{2}}
\end{array}\right)
$$

for $i, j=1,2,3$; and

$$
\begin{aligned}
& V_{R}^{14}=\frac{x_{1}^{*} \cos \theta_{R}}{m_{X}}, \quad V_{R}^{24}=\frac{x_{2}^{*} \cos \theta_{R}}{m_{X}}, \\
& V_{R}^{34}=\sin \theta_{R}-\frac{\left(m_{t^{\prime}}^{2}+m_{t}^{2}\right)\left(\left|x_{1}\right|^{2}+\left|x_{2}\right|^{2}\right) \cos ^{2} \theta_{R} \sin \theta_{R}}{2\left(m_{t^{\prime}}^{2}-m_{t}^{2}\right) m_{X}^{2}} \\
& V_{R}^{41}=-\frac{x_{1}}{m_{X}}, \quad V_{R}^{42}=-\frac{x_{2}}{m_{X}}, \\
& V_{R}^{43}=-\sin \theta_{R}+\frac{\left(\left|x_{1}\right|^{2}+\left|x_{2}\right|^{2}\right)\left(3 m_{t^{\prime}}^{2}-m_{t}^{2}+\left(m_{t^{\prime}}^{2}+m_{t}^{2}\right) \cos 2 \theta_{R}\right) \sin \theta_{R}}{4\left(m_{t^{\prime}}^{2}-m_{t}^{2}\right) m_{X}^{2}}, \\
& V_{R}^{44}=\cos \theta_{R}-\frac{\left(\left|x_{1}\right|^{2}+\left|x_{2}\right|^{2}\right)\left(m_{t^{\prime}}^{2}-3 m_{t}^{2}-\left(m_{t^{\prime}}^{2}+m_{t}^{2}\right) \cos 2 \theta_{R}\right) \cos \theta_{R}}{4\left(m_{t^{\prime}}^{2}-m_{t}^{2}\right) m_{X}^{2}} .
\end{aligned}
$$

The masses, at order $\epsilon^{2}$, are

$$
\begin{aligned}
& m_{u}^{2}=\tilde{m}_{u}^{2}, \quad m_{c}^{2}=\tilde{m}_{c}^{2} \\
& m_{t}^{2}=m_{1, t}^{2}+\frac{\left(\left|x_{1}\right|^{2}+\left|x_{2}\right|^{2}\right) m_{1, t}^{2}}{m_{X}^{2}} \sin ^{2} \theta_{R} \\
& m_{t^{\prime}}^{2}=m_{1, t^{\prime}}^{2}+\frac{\left(\left|x_{1}\right|^{2}+\left|x_{2}\right|^{2}\right) m_{1, t^{\prime}}^{2}}{m_{X}^{2}} \cos ^{2} \theta_{R}
\end{aligned}
$$

where $m_{1, t}$ and $m_{1, t^{\prime}}$ are the top and top prime masses in the 1 generation case, eq. (2.5).

A similar procedure can be followed for $V_{L}$ :

$$
V_{L}^{i j}=\left(\begin{array}{ccc}
1 & -\frac{x_{1}^{*} x_{2} m_{c} m_{u}}{\left(m_{c}^{2}-m_{u}^{2}\right) m_{X}^{2}} & -\frac{x_{1}^{*} m_{u} \sin \theta_{L}}{m_{t}^{2}} \\
\frac{x_{1} x_{2}^{*} m_{c} m_{u}}{\left(m_{c}^{2}-m_{u}^{2}\right) m_{X}^{2}} & 1 & -\frac{x_{2}^{*} m_{c} \sin \theta_{L}}{m_{t}^{2}} \\
\frac{x_{1}\left(m_{t^{\prime}}^{2}-m_{t}^{2}\right) m_{u} \cos \theta_{L} \sin \theta_{L}}{m_{t^{\prime}}^{2} m_{t}^{2}} & \frac{x_{2}\left(m_{t^{\prime}}^{2}-m_{t}^{2}\right) m_{c} \cos \theta_{L} \sin \theta_{L}}{m_{t^{\prime}}^{2} m_{t}^{2}} & \cos \theta_{L}+\frac{\left(\left|x_{1}\right|^{2}+\left|x_{2}\right|^{2}\right) \cos \theta_{L} \sin ^{2} \theta_{L}}{\left(m_{t^{\prime}}^{2}-m_{t}^{2}\right)}
\end{array}\right)
$$


with $i, j=1,2,3$; and

$$
\begin{array}{rlrl}
V_{L}^{14}=\frac{x_{1}^{*} m_{u} \cos \theta_{L}}{m_{t^{\prime}}^{2}}, & V_{L}^{24}=\frac{x_{2}^{*} m_{c} \cos \theta_{L}}{m_{t^{\prime}}^{2}}, & V_{L}^{34}=\sin \theta_{L}-\frac{\left(\left|x_{1}\right|^{2}+\left|x_{2}\right|^{2}\right) \cos ^{2} \theta_{L} \sin \theta_{L}}{\left(m_{t^{\prime}}^{2}-m_{t}^{2}\right)}, \\
V_{L}^{41}=-\frac{x_{1} m_{u}}{m_{X}^{2}}, & V_{L}^{42}=-\frac{x_{2} m_{c}}{m_{X}^{2}}, & V_{L}^{43} & =-\sin \theta_{L}+\frac{\left(\left|x_{1}\right|^{2}+\left|x_{2}\right|^{2}\right) \cos ^{2} \theta_{L} \sin \theta_{R}}{m_{t^{\prime}}^{2}-m_{t}^{2}}, \\
V_{L}^{44} & =\cos \theta_{L}+\frac{\left(\left|x_{1}\right|^{2}+\left|x_{2}\right|^{2}\right) \cos \theta_{L} \sin ^{2} \theta_{L}}{m_{t^{\prime}}^{2}-m_{t}^{2}}
\end{array}
$$

with mass eigenstates

$$
\begin{aligned}
& m_{u}^{2}=\tilde{m}_{u}^{2}, \quad m_{c}^{2}=\tilde{m}_{c}^{2}, \\
& m_{t}^{2}=m_{1, t}^{2}+\left(\left|x_{1}\right|^{2}+\left|x_{2}\right|^{2}\right) \sin ^{2} \theta_{L}, \\
& m_{t^{\prime}}^{2}=m_{1, t^{\prime}}^{2}+\left(\left|x_{1}\right|^{2}+\left|x_{2}\right|^{2}\right) \cos ^{2} \theta_{L} .
\end{aligned}
$$

The expressions for the masses coincide with the ones found from the right-handed mixing once the relations between $\theta_{L}$ and $\theta_{R}$ are taken into account.

\subsection{Charged gauge boson: $W^{ \pm}$couplings and CKM matrix}

In the basis we discussed above (gauge interaction basis), the couplings of the $W^{ \pm}$bosons is given by a 3 by 4 flavour matrix in the form:

$$
\mathcal{L}_{W^{ \pm}}=\frac{g}{\sqrt{2}}\left(\bar{u}_{L}, \bar{c}_{L}, \bar{t}_{L}, \bar{U}_{L}\right) \cdot\left(\begin{array}{ccc}
1 & & \\
& 1 & \\
& & 1 \\
0 & 0 & 0
\end{array}\right) \cdot \gamma^{\mu}\left(\begin{array}{l}
d_{L} \\
s_{L} \\
b_{L}
\end{array}\right) W_{\mu}^{+}+\text {h.c. }
$$

where the new fermion $U$ does not couple to the down-type quarks. In the mass eigenstate basis, the mixing in the left-handed sector will generate couplings with the heavy $t^{\prime}$, and, in matricial form, the coupling can be written as

$$
g_{W L}^{I j}=\frac{g}{\sqrt{2}} V_{C K M}^{I j}=\frac{g}{\sqrt{2}} V_{L}^{\dagger} \cdot\left(\begin{array}{ccc} 
& & \\
\tilde{V}_{C K M} & \\
0 & 0 & 0
\end{array}\right) .
$$

The upper 3 by 3 block, that describes the couplings of the standard quarks, is given by the matrix

$$
V_{3, C K M}^{i j}=V_{C K M}^{i j}=\sum_{l=1}^{3} V_{L}^{*, l i} \tilde{V}_{C K M}^{l j},
$$

that corresponds to the CKM matrix as measured by tree level SM processes. From this equation we can see that the measured CKM matrix is not equal to the misalignment between the two standard Yukawa matrices; however, this is approximately true because 
the mixing angles in $V_{L}$ are small. The couplings of the heavy top to the SM down quarks is described by a 3-component vector

$$
V_{C K M}^{t^{\prime} j}=\sum_{l=1}^{3} V_{L}^{*, l 4} \tilde{V}_{C K M}^{l j} .
$$

Note that in this model the 3 by 3 CKM matrix is not unitary. In fact

$$
\begin{aligned}
& {\left[V_{3, C K M} \cdot V_{3, C K M}^{\dagger}\right]^{i j}=\delta^{i j}-V_{L}^{*, 4 i} V_{L}^{4 j},} \\
& {\left[V_{3, C K M}^{\dagger} \cdot V_{3, C K M}\right]^{i j}=\delta^{i j}-V_{C K M}^{*, t^{\prime} i} V_{C K M}^{t^{\prime} j} ;}
\end{aligned}
$$

where we have used the unitarity of $\tilde{V}_{C K M}$ and $V_{L}$. The violation of unitarity are always proportional to the matrix elements $V_{L}^{4 i}$, which are related to the couplings of the $t^{\prime}$ to the $W$.

\subsection{Neutral gauge boson: $Z^{0}$ couplings and FCNCs}

In this model, the couplings of the $Z^{0}$ will also develop off-diagonal terms, absent in the SM, therefore giving rise to tree-level flavour changing neutral currents (FCNC). Note however that the FCNCs will uniquely involve up-type quarks, therefore the strong bounds from Kaon and B meson mixing are avoided. Nevertheless, as we will see, the measurements in the $\mathrm{D}$ meson sector are yet constraining. Let's first recall that the couplings of the $Z$ to SM up quarks are proportional to $\frac{g}{c_{W}}\left(\frac{1}{2}-\frac{2}{3} s_{W}^{2}\right)$ for a left-handed up, and $\frac{g}{c_{W}}\left(-\frac{2}{3} s_{W}^{2}\right)$ for a right-handed up (where as usual $s_{W}$ and $c_{W}$ indicate respectively the sine and cosine of the Weinberg angle); while for the new $U$ quark the couplings are proportional to

$$
\frac{g}{c_{W}}\left(-\frac{1}{2}-\frac{2}{3} s_{W}^{2}\right)
$$

for both left- and right-handed components. In terms of interaction eigenstates, the lefthanded couplings of the $Z$ can therefore be written as

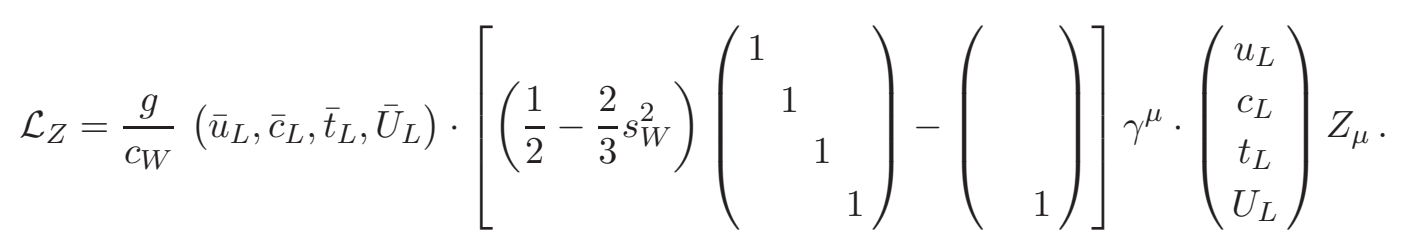

In the mass eigenstate basis, the coupling becomes

$$
g_{Z L}^{I J}=\frac{g}{c_{W}}\left(\frac{1}{2}-\frac{2}{3} s_{W}^{2}\right) \delta^{I J}-\frac{g}{c_{W}} V_{L}^{*, 4 I} V_{L}^{4 J}
$$

where $I, J=1,2,3,4$. Again, the flavour violation is governed by $V_{L}^{4 i}$ elements! Analogously for the right-handed couplings we obtain

$$
g_{Z R}^{I J}=\frac{g}{c_{W}}\left(-\frac{2}{3} s_{W}^{2}\right) \delta^{I J}-\frac{1}{2} \frac{g}{c_{W}} V_{R}^{*, 4 I} V_{R}^{4 J} .
$$




\subsection{Higgs boson couplings}

Finally, for the Higgs, in the interaction basis

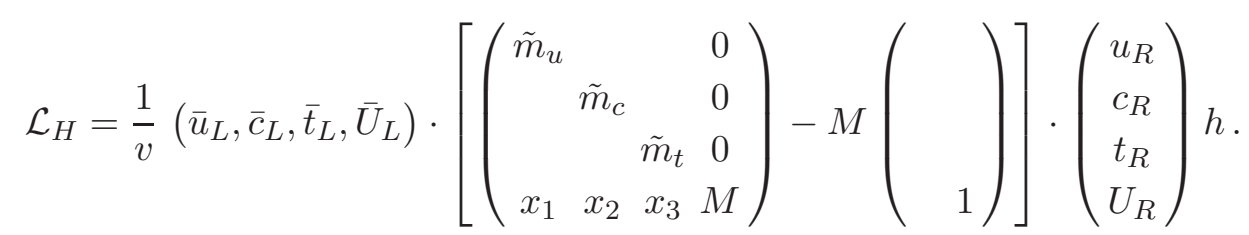

The first term reproduces the mass matrix, therefore it will be diagonal in the mass eigenstate basis: the flavour violation is generated by the second term with an entry only in the 44 term, similar to the $Z$ couplings. In the mass eigenstate basis the coupling reads:

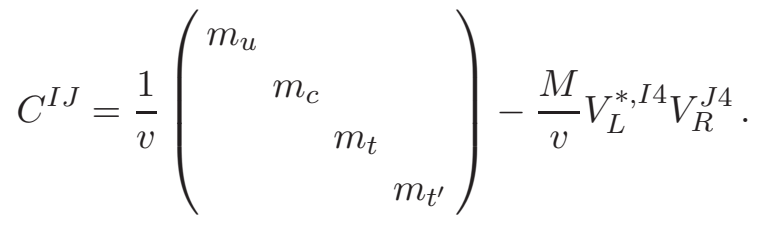

\section{Tree level bounds}

The inclusion of a non-standard doublet of quarks generates a richer flavour structure than the SM one: we have seen a non-unitary CKM matrix and flavour violating couplings of the $Z$ and Higgs in the up sector. In this section we discuss tree level bounds on the new parameters, mainly coming from electroweak precision tests at accelerators, low energy observables as the weak charge of atoms, and flavour bounds coming from D meson processes. Due to the absence of modifications of the coupling of the down quarks to the $Z$ and Higgs, therefore the absence of FCNCs in the down sector, the precise measurements in the Kaon and B meson sector do not pose very tight bounds. Such bounds may arise at loop level, via the modification of the CKM matrix and the new CP violating phases, and these effects will be briefly discussed in the next section.

\subsection{Precision electroweak measurements}

In [14] we imposed the bounds on the top sector from precisely measured quantities in the SM. For earlier bounds see for example $[17,18]$. We considered deviations on the $Z b \bar{b}$ coupling, which are absent in this specific case. We have used the exact formulas in [19] to check that the one-loop contributions to the $Z b \bar{b}$ coupling are present but small for our non-standard doublet. Note that this is true for our specific non-standard doublet, while for other cases, as for example a singlet, these bounds are relevant. We studied the deviations in the $W t b$ couplings where we allow at most a $20 \%$ deviation from 1 , electroweak precision tests (in particular the $T$ parameter) where we allow $-0.1<T<0.4$ (here we assume $m_{h}=120 \mathrm{GeV}$ and vanishing $S$ and $U$ ), finally the direct bound from Tevatron. The latter bound is nominally at $311 \mathrm{GeV}$, however the experiments only look at the $t^{\prime} \rightarrow W b$ decay mode, therefore we pose the bound until the branching ratio drops significantly below one: in other words, the bound is valid for $m_{t^{\prime}}<290 \mathrm{GeV}$. In the left panel of figure 1 , we show the allowed region in the $m_{t^{\prime}}-\sin \theta_{R}^{u}$ parameter space. Note that 

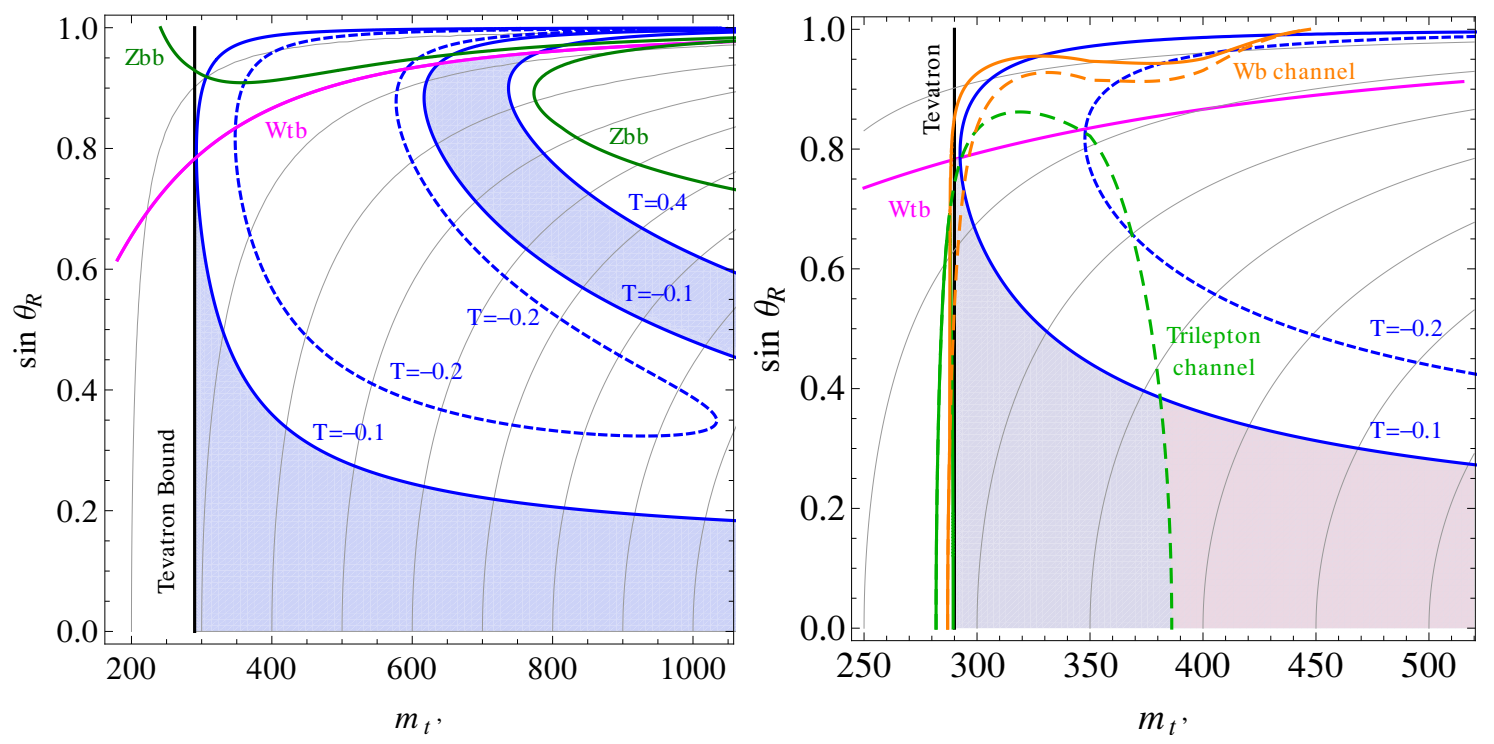

Figure 1. Bounds on the top mixing as a function of $m_{t^{\prime}}$ and $\sin \theta_{R}^{u}$. In magenta $W t b$, in green $Z b b$, in black the direct bound from Tevatron, in blue the bound from the $T$ parameter. The grey lines correspond to fixed values of $M=m_{X}$. The light blue areas are the allowed regions. On the right panel, we show the bounds from CMS in the trilepton channel and $W b$ channel. The dashed lines correspond to the case of a decoupled Higgs, the solid lines to $m_{h}=115 \mathrm{GeV}$. The coloured areas are the allowed regions.

the upper region on the right corresponds to value of the $\lambda$ Yukawa coupling close to the non-perturbative region, therefore non-perturbative effects should be considered and the formulas we use are not reliable in the upper right corner of the left panel in figure 1.

\subsection{Direct bounds at the LHC (CMS)}

The CMS collaboration recently published new bounds on a heavy $t^{\prime}$ with the 2011 data collected by the LHC. They consider both the channel $W b$ and the channel $Z t$, in both cases assuming pair production and $100 \%$ branching ratios. Here we will use their results to pose bounds on our case, where all decay channels are open.

For the $t Z$ channel [22], they select events with a pair of leptons (electrons or muons) near the $Z$ mass (the window for the dilepton invariant mass is set between 60 and $120 \mathrm{GeV}$ ), one additional lepton and at least two additional jets. Their analysis is limited to a counting experiment and the bound is extracted by comparing the expected number of events from backgrounds and signal to the observed one: the preliminary results, based on an integrated luminosity of $191 \mathrm{pb}^{-1}$ shows that there are no events in the dataset. In our case, many different decay chains can give rise to events that pass the event selection used by CMS:

$$
\begin{aligned}
p p & \rightarrow\left(t^{\prime} \rightarrow Z t\right)\left(\bar{t}^{\prime} \rightarrow Z \bar{t}\right) \rightarrow b \bar{b} Z Z W^{+} W^{-} \\
& \rightarrow\left(t^{\prime} \rightarrow Z t\right)\left(\bar{t}^{\prime} \rightarrow W^{+} \bar{b}\right) \rightarrow b \bar{b} Z W^{+} W^{-} \\
& \rightarrow\left(t^{\prime} \rightarrow W^{-} b\right)\left(\bar{t}^{\prime} \rightarrow Z \bar{t}\right) \rightarrow b \bar{b} Z W^{+} W^{-} \\
& \rightarrow\left(t^{\prime} \rightarrow Z t\right)\left(\bar{t}^{\prime} \rightarrow h \bar{t}\right) \rightarrow b \bar{b} b \bar{b} Z W^{+} W^{-} \\
& \rightarrow\left(t^{\prime} \rightarrow h t\right)\left(\bar{t}^{\prime} \rightarrow Z \bar{t}\right) \rightarrow b \bar{b} b \bar{b} Z W^{+} W^{-}
\end{aligned}
$$


where one of the $Z$ 's and one of the $W$ 's decay leptonically. In extracting the bound, we consider all these channels together, under the assumptions that the efficiency of the cuts is the same: this is not necessarily true, because the kinematics of the event are different, and we plan to refine this analysis in a future work. The final result is shown on the right panel in figure 1: the dark green area is excluded for a Higgs mass of $m_{h}=115 \mathrm{GeV}$, while the lighter green one is excluded in the decoupled Higgs limit, where the absence of the $t H$ decay mode boosts the $t Z$ branching.

The analysis in the $W b[23]$ is more refined because of the presence of an irreducible background from $t \bar{t}$ : the selection criteria are the presence of an isolated lepton (electron or muon), a missing $p_{T}$ greater than $20 \mathrm{GeV}$, at least 4 jets where one or more are tagged as b-jets. To distinguish the $t \bar{t}$ background, the collaboration reconstructs the invariant mass of the top or $t^{\prime}$ under the assumption of the topology

$$
p p \rightarrow\left(t / t^{\prime} \rightarrow W^{+} b\right)\left(\bar{t} / \bar{t}^{\prime} \rightarrow W^{-} \bar{b}\right) \rightarrow b \bar{b} l^{ \pm} \nu j j,
$$

and fits the distribution of backgrounds and signal with a free mass for the $t^{\prime}$. In the preliminary results, an integrated luminosity of $573 \mathrm{pb}^{-1}$ for electrons and $821 \mathrm{pb}^{-1}$ has been considered. In our case different decay chains can give rise to events that pass the selection rules of this analysis. However, the reconstruction algorithm will not work on events with decays to $Z t$ and $h t$, where two extra jets or missing energy is coming from the decay of the $Z$ and $h$. A more detailed study would be required to take into account such events. To be conservative, therefore, we will limit ourselves to rescale the limit by the branching ratio $\mathrm{BR}\left(t^{\prime} \rightarrow W b\right)$ : the result is shown in figure 1, where the dark (light) grey area correspond to a Higgs mass of $m_{h}=115 \mathrm{GeV}$ (1000 GeV - decoupled limit). Note that for low $\sin \theta_{R}$, the bound is $m_{t^{\prime}} \gtrsim 290 \mathrm{GeV}$, which is the same bound we assumed for the Tevatron data and given by the suppression in the $W b$ branching ratio.

The plot also shows that the $Z t$ or trilepton channel is much more promising than the more traditional $W b$ one, also taking into account the more limited luminosity of the preliminary data. Note also that a trilepton signature will also arise the from single production of $t^{\prime}$, as we will discuss in section 5, and that such events are not taken into account in extracting this bound.

\section{3 $\quad D_{0}-\bar{D}_{0}$ mixing and $D_{0} \rightarrow l^{+} l^{-}$decays}

In our model, FCNCs will only affect the up quark sector, therefore the most severe bounds will come from the $D$ meson sector. The most recent experimental measurements in the $D_{0}-\bar{D}_{0}$ mixing (with no CP violation) are [24]

$$
x_{D}=\frac{\Delta m_{D}}{\Gamma_{D}}=0.0100_{-0.0026}^{+0.0024}, \quad y_{D}=\frac{\Delta \Gamma_{D}}{2 \Gamma_{D}}=0.0076_{-0.0018}^{+0.0017} .
$$

In the SM, $x_{D}$ is dominated by long distance effects, and its value cannot be reliably estimated. Therefore, it is possible that the new physics saturates the experimental value and this situation will provide the most conservative bound on the new contributions.

Another potential bound may arise from the dilepton $\Delta C=1$ decays: these channels have not been measured, and the present experimental limits are [13]:

$$
B R\left(D_{0} \rightarrow e^{+} e^{-}\right) \exp <1.2 \times 10^{-6}, \quad B R\left(D_{0} \rightarrow \mu^{+} \mu^{-}\right) \exp <1.3 \times 10^{-6} .
$$


Contrary to the $\Delta C=2$ contributions to the mixing, the branching ratio can be calculated and, in the SM, one obtains

$$
B R\left(D_{0} \rightarrow \mu^{+} \mu^{-}\right)_{\mathrm{SM}} \sim 3 \times 10^{-13},
$$

while the decay into electrons is suppressed by the electron mass square. In both cases, therefore, the SM prediction lies well below the experimental limit.

In our case, the contribution of new physics is given in both cases by a tree level exchange of a $Z$ boson in the s-channel:

$$
c \bar{u} \rightarrow Z^{*} \rightarrow u \bar{c}, \quad \text { and } \quad c \bar{u} \rightarrow Z^{*} \rightarrow l^{+} l^{-} .
$$

We will only consider the right-handed couplings, because the left-handed ones are suppressed by extra powers of the light quark masses. The contribution of the new physics can be written as:

$$
\begin{gathered}
\delta x_{D}=\frac{f_{D}^{2} m_{D} B_{D}}{2 m_{Z}^{2} \Gamma_{D}} \frac{2}{3} r\left(m_{c}, m_{Z}\right)\left(g_{Z R}^{u c}\right)^{2}, \\
\delta B R\left(D_{0} \rightarrow l^{+} l^{-}\right)=\frac{f_{D}^{2} m_{D} m_{l}^{2}}{32 \pi m_{Z}^{4} \Gamma_{D}} \sqrt{1-\frac{4 m_{l}^{2}}{m_{D}^{2}}} \frac{\pi \alpha}{\cos ^{2} \theta_{W} \sin ^{2} \theta_{W}}\left(g_{Z R}^{u c}\right)^{2},
\end{gathered}
$$

where the explanation of the formula and the definitions and numerical values of the various parameters can be found in [24]. If we assume that the new physics saturates the value of $x_{D}$, we can predict the value for the branching ratio; this value will become an upper bound in the case in which the mixing is dominated by the SM:

$$
B R\left(D_{0} \rightarrow l^{+} l^{-}\right)_{\mathrm{NPh}}=\frac{3}{32} \frac{\alpha}{\cos ^{2} \theta_{W} \sin ^{2} \theta_{W}} \frac{x_{D}}{B_{D} r\left(m_{c}, m_{Z}\right)} \frac{m_{l}^{2}}{m_{Z}^{2}} \sqrt{1-\frac{4 m_{l}^{2}}{m_{D}^{2}}} .
$$

Plugging in numbers, at one sigma, we find

$$
B R\left(D_{0} \rightarrow \mu^{+} \mu^{-}\right)_{\mathrm{NPh}}=(0.8 \pm 0.2) \times 10^{-10},
$$

therefore we are still far from the experimental bound. For electrons, the branching is suppressed by a factor $\left(m_{e} / m_{\mu}\right)^{2}$.

The most significant constraint from new physics, therefore, comes from $x_{D}$. In our case,

$$
\left(g_{Z R}^{u c}\right)^{2}=\frac{\pi \alpha}{\cos ^{2} \theta_{W} \sin ^{2} \theta_{W}}\left|V_{R}^{41}\right|^{2}\left|V_{R}^{42}\right|^{2} .
$$

To fit the experimental value would require

$$
\left|V_{R}^{41}\right|\left|V_{R}^{42}\right|=2.44_{-0.34}^{+0.28} \times 10^{-4} .
$$

This implies a bound at $3 \sigma,^{1}$

$$
\left|V_{R}^{41}\right|\left|V_{R}^{42}\right|<3.2 \times 10^{-4} .
$$

As we will see in the following, this is the most stringent bound on flavour violation.

\footnotetext{
${ }^{1}$ This bound roughly agrees with the results in [25], where effective operators are considered. The relevant parameter, as defined in [25], is $\left|\tilde{z}_{1}\right|<5.7 \times 10^{-7}\left(\frac{\Lambda}{1 \mathrm{TeV}}\right)^{2}$, where $\Lambda$ is the scale suppressing the operator $\left(\Lambda=m_{Z}\right.$ in our case). Converting the bound in our parameterisation, we obtain $\left|V_{R}^{41}\right|\left|V_{R}^{42}\right|<2.0 \times 10^{-4}$.
} 


\begin{tabular}{|c|c|c|c|c|}
\hline mode & SM short & SM long & our model & exp. limit \\
\hline$D^{+} \rightarrow \pi^{+} e^{+} e^{-}$ & $9.4 \times 10^{-9}$ & $1.0 \times 10^{-6}$ & $c \rightarrow u l^{+} l^{-}$ & $7.4 \times 10^{-6}$ \\
\hline$D^{+} \rightarrow \pi^{+} \mu^{+} \mu^{-}$ & $9.4 \times 10^{-9}$ & $1.0 \times 10^{-6}$ & $c \rightarrow u l^{+} l^{-}$ & $3.9 \times 10^{-6}$ \\
\hline$D^{+} \rightarrow \rho^{+} \mu^{+} \mu^{-}$ & $4.8 \times 10^{-9}$ & $1.5 \div 1.8 \times 10^{-6}$ & $c \rightarrow u l^{+} l^{-}$ & $5.6 \times 10^{-4}$ \\
\hline$D_{0} \rightarrow \gamma \gamma$ & & & 1-loop & $2.7 \times 10^{-5}$ \\
\hline$D_{0} \rightarrow \pi^{0} e^{+} e^{-}$ & $1.9 \times 10^{-9}$ & $2.1 \times 10^{-7}$ & $c \rightarrow u l^{+} l^{-}$ & $4.5 \times 10^{-5}$ \\
\hline$D_{0} \rightarrow \pi^{0} \mu^{+} \mu^{-}$ & $1.9 \times 10^{-9}$ & $2.1 \times 10^{-7}$ & $c \rightarrow u l^{+} l^{-}$ & $1.8 \times 10^{-4}$ \\
\hline$D_{0} \rightarrow \eta e^{+} e^{-}$ & $2.5 \times 10^{-10}$ & $4.9 \times 10^{-8}$ & $c \rightarrow u l^{+} l^{-}$ & $1.1 \times 10^{-4}$ \\
\hline$D_{0} \rightarrow \eta \mu^{+} \mu^{-}$ & $2.5 \times 10^{-10}$ & $4.9 \times 10^{-8}$ & $c \rightarrow u l^{+} l^{-}$ & $5.3 \times 10^{-4}$ \\
\hline$D_{0} \rightarrow \pi^{+} \pi^{-} e^{+} e^{-}$ & & & $c \rightarrow u l^{+} l^{-}$ & $3.73 \times 10^{-4}$ \\
\hline$D_{0} \rightarrow \rho^{0} e^{+} e^{-}$ & & & $c \rightarrow u l^{+} l^{-}$ & $1.0 \times 10^{-4}$ \\
\hline$D_{0} \rightarrow \pi^{+} \pi^{-} \mu^{+} \mu^{-}$ & & & $c \rightarrow u l^{+} l^{-}$ & $3.0 \times 10^{-5}$ \\
\hline$D_{0} \rightarrow \rho^{0} \mu^{+} \mu^{-}$ & $9.7 \times 10^{-10}$ & $3.5 \div 4.7 \times 10^{-7}$ & $c \rightarrow u l^{+} l^{-}$ & $2.2 \times 10^{-5}$ \\
\hline$D_{0} \rightarrow \omega e^{+} e^{-}$ & & & $c \rightarrow u l^{+} l^{-}$ & $1.8 \times 10^{-4}$ \\
\hline$D_{0} \rightarrow \omega \mu^{+} \mu^{-}$ & $9.1 \times 10^{-10}$ & $3.3 \div 4.5 \times 10^{-7}$ & $c \rightarrow u l^{+} l^{-}$ & $8.3 \times 10^{-4}$ \\
\hline$D_{0} \rightarrow K^{+} K^{-} e^{+} e^{-}$ & & & $c \rightarrow u l^{+} l^{-}$ & $3.15 \times 10^{-4}$ \\
\hline$D_{0} \rightarrow \phi e^{+} e^{-}$ & & & & $5.2 \times 10^{-5}$ \\
\hline$D_{0} \rightarrow K^{+} K^{-} \mu^{+} \mu^{-}$ & & & $c \rightarrow u l^{+} l^{-}$ & $3.3 \times 10^{-5}$ \\
\hline$D_{0} \rightarrow \phi \mu^{+} \mu^{-}$ & 0 & $6.5 \div 9 \times 10^{-8}$ & & $3.1 \times 10^{-5}$ \\
\hline$D_{0} \rightarrow \pi^{+} K^{-} e^{+} e^{-}$ & & & & $3.85 \times 10^{-4}$ \\
\hline$D_{0} \rightarrow \pi^{+} K^{-} \mu^{+} \mu^{-}$ & & & & $3.59 \times 10^{-4}$ \\
\hline$D_{0} \rightarrow \pi^{+} \pi^{-} \pi^{0} e^{+} e^{-}$ & & & $c \rightarrow u l^{+} l^{-}$ & $8.1 \times 10^{-4}$ \\
\hline$D_{s}^{+} \rightarrow K^{+} \mu^{+} \mu^{-}$ & $9.0 \times 10^{-10}$ & $4.3 \times 10^{-8}$ & $c \rightarrow u l^{+} l^{-}$ & $3.6 \times 10^{-5}$ \\
\hline$D_{s}^{+} \rightarrow K^{+} e^{+} e^{-}$ & $9.0 \times 10^{-10}$ & $4.3 \times 10^{-8}$ & $c \rightarrow u l^{+} l^{-}$ & $1.6 \times 10^{-3}$ \\
\hline$D_{s}^{+} \rightarrow K^{*}(892)^{+} \mu^{+} \mu^{-}$ & $1.6 \times 10^{-9}$ & $5 \div 7 \times 10^{-7}$ & $c \rightarrow u l^{+} l^{-}$ & $1.4 \times 10^{-3}$ \\
\hline
\end{tabular}

Table 1. Rare decays of D mesons with SM short and long distance contributions, the decay channel within the vector-like model and the corresponding experimental figures.

\subsection{Rare decays of $D$ mesons}

Many rare decays of the $D$ mesons via FCNC have been tested and have strong bounds from experiments such as CLEO and BES. In the SM they are loop generated and often dominated by long distance effects while, in our model, they may receive tree level contributions from the $Z$ exchange. A list of the experimental limits [13], standard model predictions from short and long distance [26] calculations and the possible contribution in our case is presented in table 1 . The short distance SM result is dominated by the operator $\mathcal{O}_{9}[27]$

$$
\mathcal{L}=-\frac{4 G_{F}}{\sqrt{2}} V_{c s}^{*} V_{u s} \frac{\alpha}{4 \pi} c_{9} \bar{u} \gamma^{\mu} P_{L} c \bar{l} \gamma_{\mu} l
$$

where $c_{9}\left(m_{W}\right) \sim \frac{4}{9} \log \frac{m_{s}}{m_{d}}=1.34$, while the contribution of the penguins, enhanced by QCD corrections is small. 
In our model, the right-handed couplings of the $Z$ generate the operators $\mathcal{O}_{9}^{\prime}$ and $\mathcal{O}_{10}^{\prime}[27]$, with coefficients

$$
\mathcal{L}=\frac{G_{F}}{\sqrt{2}} V_{R}^{* 41} V_{R}^{42} \bar{u} \gamma^{\mu} P_{R} c\left\{\left(1-4 \sin ^{2} \theta_{W}\right) \bar{l} \gamma_{\mu} l-\bar{l} \gamma_{\mu} \gamma_{5} l\right\}
$$

Those operators contribute to the short distance term, they do not significantly affect the long distance one because the pair of leptons comes from an heavy $Z$ boson that do not mix significantly to light vector mesons. The main difference between our contributions and the $\mathrm{SM}$ one is that in our case right-handed quarks are involved, and the form factors are not precisely known. Here we will simply assume that the form factor for right-handed and lefthanded quarks are the same to extract an order of magnitude estimate of the contribution. Under such assumption, we can calculate the ratio $\xi_{D}$ between the new physics and SM contribution (where the form factors cancel out) and obtain

$$
\xi_{D}=\frac{2 \pi^{2}\left(1-4 \sin ^{2} \theta_{W}+8 \sin ^{4} \theta_{W}\right)}{\alpha^{2}\left|V_{c s}\right|^{2}\left|V_{u s}\right|^{2} c_{9}^{2}}\left|V_{R}^{41}\right|^{2}\left|V_{R}^{42}\right|^{2}=0.5 \times 10^{6} \times\left|V_{R}^{41}\right|^{2}\left|V_{R}^{42}\right|^{2} .
$$

This enhancement factor allows some of the bounds to be relevant for our model. However, imposing the $D_{0}-\bar{D}_{0}$ mixing bound, we obtain that

$$
\xi_{D}<0.05
$$

therefore those processes should not impose further bounds.

\subsection{Top quark FCNC rare decays: $t \rightarrow Z c, Z u$}

FCNCs mediated by the $Z$ boson can also affect the physics of the top quark. The only observable effect is in rare decays of the top quark: $t \rightarrow Z c$ and $t \rightarrow Z u$, which are very suppressed and induced by loops in the SM. The present bound on the FCNC decays of the top is $[28,29]$ :

$$
\frac{\Gamma(t \rightarrow Z c)+\Gamma(t \rightarrow Z u)}{\Gamma(t \rightarrow W b)}<3.3 \%
$$

which involves the partial width in $W b$ that has not been measured yet. In our case, there is a tree level vertex of the $Z$ with top and a light quark. The widths can be written as $[30,31]$ (neglecting the up and charm masses)

$$
\begin{aligned}
\Gamma(t \rightarrow Z q) & =\left(g_{Z R}^{t q}\right)^{2} \frac{m_{t}^{3}}{8 \pi m_{Z}^{2}} f\left(\frac{m_{Z}}{m_{t}}\right), \\
\Gamma(t \rightarrow W b) & =\frac{G_{F} m_{t}^{2}}{8 \pi \sqrt{2}}\left|V_{t b}\right|^{2} f\left(\frac{m_{W}}{m_{t}}\right) ;
\end{aligned}
$$

where

$$
f(x)=\left(1-x^{2}\right)^{2}\left(1+2 x^{2}\right)
$$

In our model,

$$
\Gamma(t \rightarrow Z u)=\frac{G_{F} m_{t}^{3}}{16 \pi \sqrt{2}}\left|V_{R}^{43}\right|^{2}\left|V_{R}^{41}\right|^{2} f\left(\frac{m_{Z}}{m_{t}}\right),
$$

and similarly for $t \rightarrow Z c$ with $V_{R}^{41} \rightarrow V_{R}^{42}$. 
The bound, therefore, can be written as

$$
\frac{\left|V_{R}^{43}\right|^{2}\left(\left|V_{R}^{41}\right|^{2}+\left|V_{R}^{42}\right|^{2}\right)}{2\left|V_{t b}\right|^{2}} \frac{f\left(m_{Z} / m_{t}\right)}{f\left(m_{W} / m_{t}\right)}<3.3 \%
$$

and

$$
\left|V_{R}^{43}\right| \sqrt{\left|V_{R}^{41}\right|^{2}+\left|V_{R}^{42}\right|^{2}}<0.28\left|V_{t b}\right| .
$$

Note that this bound depends on $V_{R}^{43} \sim \sin \theta_{R}^{u}$, and on the value of $V_{t b}$ which can deviate from one and therefore make the bound stronger. Even though this bound is much milder that the one from the $D_{0}$ mesons, the different dependence on the two mixing angles $V_{R}^{41}$ and $V_{R}^{42}$ makes it relevant. These rare decay modes of the top quark may be relevant for LHC with high statistics and can be studied in detail at an electron-positron linear collider.

\subsection{Atomic parity violation}

Another strong bound comes from the modifications of the couplings of the up with the $Z^{0}$ boson: experiments can measure the weak charge $Q_{W}$ of the nucleus. The most precise test is from atomic parity violation in Cesium ${ }^{133} \mathrm{Cs}$ [13]:

$$
\left.Q_{W}\left({ }^{133} \mathrm{Cs}\right)\right|_{\text {exp. }}=-73.20 \pm 0.35,\left.\quad Q_{W}\left({ }^{133} \mathrm{Cs}\right)\right|_{\mathrm{SM}}=-73.15 \pm 0.02
$$

In general, the weak charge can be written as [32]

$$
Q_{W}=(2 Z+N)\left(\tilde{g}_{Z L}^{u}+\tilde{g}_{Z R}^{u}\right)+(Z+2 N)\left(\tilde{g}_{Z L}^{d}+\tilde{g}_{Z R}^{d}\right),
$$

where the $\tilde{g}$ 's are the left- and right-handed couplings of the $Z^{0}$ divided by a factor $\frac{g}{2 \cos \theta_{W}}$, and $N$ and $Z$ are the number of neutrons and protons in the nucleus. In our case, the only large deviation appears in

$$
\tilde{g}_{Z R}^{u}=-\frac{4}{3} \sin ^{2} \theta_{W}+\delta g_{Z R}, \quad \delta g_{Z R}=-\left|V_{R}^{41}\right|^{2} .
$$

The correction to the weak charge is therefore

$$
\delta Q_{W}(\mathrm{Cs})=-(2 Z+N)\left|V_{R}^{41}\right|^{2}=-188\left|V_{R}^{41}\right|^{2},
$$

where the numerical value corresponds to ${ }^{133} \mathrm{Cs}(Z=55$ and $N=78)$. As the SM prediction is in very good agreement with the experimental value and its error is very small, we can directly compare the new physics contribution to the experimental error. At 3 sigma $\left|\delta Q_{W}\right|<(-73.15+0.06)-(-73.20-1.05)=1.16$, therefore

$$
\left|V_{R}^{41}\right|<7.8 \cdot 10^{-2} .
$$

Atomic parity violation has also been measured for the Thallium ${ }^{204} \mathrm{Tl}$, however the bound is milder. ${ }^{2}$

\footnotetext{
${ }^{2}$ This case is more favourable because of the larger atomic number $(Z=81$ and $N=123)$, however the experimental precision is lower that in Cesium [13]:

$$
\left.Q_{W}(\mathrm{Tl})\right|_{\exp .}=-116.4 \pm 3.6,\left.\quad Q_{W}(\mathrm{Tl})\right|_{\mathrm{SM}}=-116.76 \pm 0.04 ;
$$

for which the correction is $\delta Q_{W}(\mathrm{Tl})=-(2 Z+N)\left|V_{R}^{41}\right|^{2}=-285\left|V_{R}^{41}\right|^{2}$, and the bound $\left|V_{R}^{41}\right|<0.19$.
} 


\subsection{CKM matrix}

The CKM matrix has been measured very precisely, both at tree and one-loop level. Even though in our case the corrections are proportional to the suppressed mixing angles in the left-handed sector, it is worth studying the detailed structure of the modified CKM matrix to ensure that no further bounds arise. In the following we will limit ourselves to tree level measurements, because loop effects will also include the effect of the heavy top $t^{\prime}$ and the flavour violating $Z$ boson couplings (a brief discussion of loop effect is present in next section). The modified CKM matrix is given in eq. (2.24): as $V_{L}$ is close to the identity matrix, we can assume that $\tilde{V}_{C K M}$ is approximately equal to the measured matrix, while $V_{L}$ generates small corrections. For a study discussing the CKM structure in the case of an iso-singlet see [33]. In this section, we will estimate how small such corrections can be.

We first observe that both $\tilde{V}_{C K M}$ and $V_{L}$ are hierarchical matrices, as the off diagonal entries of the latter are suppressed by ratios of quark masses. Therefore, we can use the Cabibbo angle $\lambda=\sin \theta_{12}$ as an expansion parameter for both (knowing that $\frac{m_{c}}{m_{t}} \sim \mathcal{O}\left(\lambda^{3}\right)$ and $\left.\frac{m_{u}}{m_{t}} \sim \mathcal{O}\left(\lambda^{7}\right)\right)$. More details about this expansion are presented in appendix A. On top of the suppression from the light quark masses, off diagonal $V_{L}$ terms are also suppressed by the new yukawa couplings $x_{i}$, which are constrained to be small as we discusses previously. One can relate the elements of $V_{L}$ that enter in the definition of $V_{C K M}$ to $V_{R}^{14}$ and $V_{R}^{24}$, and use the bounds in eq.s (3.11), (3.22) and (3.27) to find an upper bound. For instance

$$
V_{L}^{12}=\frac{m_{c} m_{u}}{m_{c}^{2}-m_{u}^{2}} \frac{x_{1} x_{2}^{*}}{m_{X}^{2}}=\frac{m_{c} m_{u}}{m_{c}^{2}-m_{u}^{2}} \frac{V_{R}^{*, 41} V_{R}^{42}}{\cos ^{2} \theta_{R}}=-V_{L}^{*, 21} .
$$

Therefore, $\left|V_{L}^{12}\right|=\left|V_{L}^{21}\right|$ is suppressed by a power $\lambda^{4}$ from the quark mass ratio, but also by the product $\left|V_{R}^{41}\right|\left|V_{R}^{42}\right|<3.2 \cdot 10^{-4}$; taking $\cos \theta_{R} \sim 1$

$$
\left|V_{L}^{12}\right|<8.3 \cdot 10^{-7} \text {. }
$$

Therefore, $V_{L}^{12}$ is always highly suppressed! Regarding $V_{L}^{13}$ and $V_{L}^{31}$, we can write

$$
V_{L}^{13}=-\frac{x_{1}^{*} m_{u}}{m_{t}^{2}} \sin \theta_{L}=-\frac{m_{u}}{m_{t}} \frac{x_{1}^{*} \sin \theta_{R}}{m_{X}}=\frac{m_{u}}{m_{t}} V_{R}^{41} \sin \theta_{R} .
$$

Besides the $\lambda^{7}$ suppression from the quark masses, this element is proportional to $\left|V_{R}^{14}\right|<$ $7.8 \cdot 10^{-2}$ (from APV in Cesium); therefore, considering $\tan \theta_{R}<0.3$ as a conservative bound, we find

$$
\left|V_{L}^{13}\right|<4.2 \cdot 10^{-7}
$$

The same is true for

$$
V_{L}^{31}=\frac{m_{t^{\prime}}^{2}-m_{t}^{2}}{m_{t^{\prime}}^{2}} \cos \theta_{L}\left(-V_{L}^{*, 13}\right)
$$

Finally,

$$
V_{L}^{23}=-\frac{x_{2}^{*} m_{c}}{m_{t}^{2}} \sin \theta_{L}=-\frac{m_{c}}{m_{t}} \frac{x_{2}^{*} \sin \theta_{R}}{m_{X}}=\frac{m_{c}}{m_{t}} V_{R}^{42} \sin \theta_{R}
$$


This element is proportional to $V_{R}^{42}$ which is smaller than 0.2 from the measurement of the charm quark to the $Z$ coupling, therefore it is maximal when this bound is saturated:

$$
\left|V_{L}^{23}\right|<2.3 \cdot 10^{-3} \times\left|V_{R}^{42}\right|
$$

The same is true for

$$
V_{L}^{32}=\frac{m_{t^{\prime}}^{2}-m_{t}^{2}}{m_{t^{\prime}}^{2}} \cos \theta_{L}\left(-V_{L}^{*, 23}\right)
$$

The final comment is about $V_{L}^{33}$ : this modifies the top couplings and therefore may seriously affect the loop processes that are Glashow-Iliopoulos-Maiani (GIM) suppressed in the SM. The tree level bound coming from modifications of $V_{t b}$ have already been considered in [14], and are less competitive than bounds from the $T$ parameter. We can estimate

$$
V_{L}^{33} \sim \cos \theta_{L}=1-\frac{1}{2} \frac{m_{t}^{2}}{m_{X}^{2}} \sin ^{2} \theta_{R}
$$

The bounds from the top sector typically imply $m_{X}>2 m_{t}$ and $\sin \theta_{R}<0.3$, therefore

$$
1-V_{L}^{33}<0.01
$$

A numerical example. We have seen that corrections to the CKM matrix are typically very small. To be more concrete, here we will focus on two numerical examples to illustrate the relevance of the corrections. First, we parameterise the two phases as $x_{1}=\left|x_{1}\right| e^{i \beta_{1}}$ and $x_{2}=\left|x_{2}\right| e^{i \beta_{2}}$. As a benchmark mass for the $t^{\prime}$ we consider $m_{t^{\prime}}=350 \mathrm{GeV}$, and a conservative bound on the mixing $\sin \theta_{R}=0.3$. As we want a maximal flavour violating effect, we will saturate all bound, in particular the strongest bound from $D_{0}-\bar{D}_{0}$ mixing $\left|V_{R}^{41}\right|\left|V_{R}^{42}\right|=3.2 \cdot 10^{-4}$. We distinguish two cases: in case A, the mixing with the up is maximal, therefore from the APV bound we fix $\left|V_{R}^{41}\right|=0.078$; in case $\mathrm{B}$, we require maximal mixing with the charm, which is bounded from top decays $\left|V_{R}^{42}\right|=0.2$. In these numerical examples only, for simplicity, we also set the phase in $\tilde{V}_{C K M}$ to zero, while the absolute values of the entries are taken from the tree level measurements in the SM [13]. The final results are listed in table 2 .

\section{Loop constraints and couplings}

So far, we have discussed tree level bounds from precision and flavour observables coming mainly from the right-handed mixing of up type quarks, while the left handed mixings are suppressed by the SM quark masses. In particular, the CKM matrix is only affected by the left-handed mixings and no significant tree level bounds arise. The situation is quite different at loop level: in fact, loops involving the modified CKM couplings, will correct the SM results. In some cases, where a precise cancellation occurs in the SM loops, even small corrections may give rise to observable effects. Another important constraint may come from $\mathrm{CP}$ violating effects, which arise in the SM from a single phase. In our case, 


\begin{tabular}{|l|c|c|c|}
\hline & $\mathrm{SM}$ & Case $\mathrm{A}$ & Case B \\
\hline$V_{C K M}^{u d}$ & 0.974 & $+1.9 \cdot 10^{-7} e^{i\left(\beta_{2}-\beta_{1}\right)}+2.6 \cdot 10^{-9} e^{-i \beta_{1}}$ & $+1.9 \cdot 10^{-7} e^{i\left(\beta_{2}-\beta_{1}\right)}+5.4 \cdot 10^{-11} e^{-i \beta_{1}}$ \\
$V_{C K M}^{u s}$ & 0.225 & $+8.5 \cdot 10^{-7} e^{i\left(\beta_{2}-\beta_{1}\right)}+1.2 \cdot 10^{-8} e^{-i \beta_{1}}$ & $+8.5 \cdot 10^{-7} e^{i\left(\beta_{2}-\beta_{1}\right)}+2.5 \cdot 10^{-10} e^{-i \beta_{1}}$ \\
$V_{C K M}^{u b}$ & 0.0039 & $+3.4 \cdot 10^{-8} e^{i\left(\beta_{2}-\beta_{1}\right)}+2.7 \cdot 10^{-7} e^{-i \beta_{1}}$ & $+3.4 \cdot 10^{-8} e^{i\left(\beta_{2}-\beta_{1}\right)}+5.6 \cdot 10^{-9} e^{-i \beta_{1}}$ \\
\hline$V_{C K M}^{c d}$ & 0.23 & $-8.1 \cdot 10^{-7} e^{-i\left(\beta_{2}-\beta_{1}\right)}+5.8 \cdot 10^{-8} e^{-i \beta_{2}}$ & $+2.8 \cdot 10^{-6} e^{-i \beta_{2}}-8.1 \cdot 10^{-7} e^{-i\left(\beta_{2}-\beta_{1}\right)}$ \\
$V_{C K M}^{c s}$ & 1.023 & $-1.9 \cdot 10^{-7} e^{-i\left(\beta_{2}-\beta_{1}\right)}+2.6 \cdot 10^{-7} e^{-i \beta_{2}}$ & $+1.3 \cdot 10^{-5} e^{-i \beta_{2}}-1.9 \cdot 10^{-7} e^{-i\left(\beta_{2}-\beta_{1}\right)}$ \\
$V_{C K M}^{c b}$ & 0.041 & $+6.1 \cdot 10^{-6} e^{-i \beta_{2}}-3.2 \cdot 10^{-9} e^{-i\left(\beta_{2}-\beta_{1}\right)}$ & $+3 \cdot 10^{-4} e^{-i \beta_{2}}-3.2 \cdot 10^{-9} e^{-i\left(\beta_{2}-\beta_{1}\right)}$ \\
\hline$V_{C K M}^{t d}$ & 0.008 & $-1 \cdot 10^{-4}-2.1 \cdot 10^{-6} e^{i \beta_{2}}-4.1 \cdot 10^{-7} e^{i \beta_{1}}$ & $-9 \cdot 10^{-5}-1.0 \cdot 10^{-4} e^{i \beta_{2}}-8.4 \cdot 10^{-9} e^{i \beta_{1}}$ \\
$V_{C K M}^{t s}$ & 0.038 & $-4.6 \cdot 10^{-4}-1 . \cdot 10^{-5} e^{i \beta_{2}}-1 . \cdot 10^{-7} e^{i \beta_{1}}$ & $-4.2 \cdot 10^{-4}-0.5 \cdot 10^{-3} e^{i \beta_{2}}-19 \cdot 10^{-10} e^{i \beta_{1}}$ \\
$V_{C K M}^{t b}$ & 0.88 & $-0.01-3.8 \cdot 10^{-7} e^{i \beta_{2}}-1.6 \cdot 10^{-9} e^{i \beta_{1}}$ & $-0.009-1.9 \cdot 10^{-5} e^{i \beta_{2}}-3.3 \cdot 10^{-11} e^{i \beta_{1}}$ \\
\hline$V_{C K M}^{t^{\prime} d}$ & & $0.0013+3.4 \cdot 10^{-6} e^{i \beta_{2}}+6.5 \cdot 10^{-7} e^{i \beta_{1}}$ & $10 \cdot 10^{-4}+1.7 \cdot 10^{-4} e^{i \beta_{2}}+13 . \cdot 10^{-9} e^{i \beta_{1}}$ \\
$V_{C K M}^{t^{\prime} s}$ & & $0.006+1.5 \cdot 10^{-5} e^{i \beta_{2}}+1.5 \cdot 10^{-7} e^{i \beta_{1}}$ & $0.006+0.7 \cdot 10^{-3} e^{i \beta_{2}}+3.1 \cdot 10^{-9} e^{i \beta_{1}}$ \\
$V_{C K M}^{t^{\prime} b}$ & & $0.14+6.1 \cdot 10^{-7} e^{i \beta_{2}}+2.6 \cdot 10^{-9} e^{i \beta_{1}}$ & $0.13+3.0 \cdot 10^{-5} e^{i \beta_{2}}+5.3 \cdot 10^{-11} e^{i \beta_{1}}$ \\
\hline
\end{tabular}

Table 2. Numerical values of the corrections to the CKM matrix for $m_{t^{\prime}}=350 \mathrm{GeV}, \sin \theta_{R}=0.3$, $\left|V_{R}^{41}\right|\left|V_{R}^{42}\right|=3.2 \cdot 10^{-4}$ and: in case $\mathrm{A},\left|V_{R}^{41}\right|=0.078$; in case $\mathrm{B},\left|V_{R}^{42}\right|=0.2$.
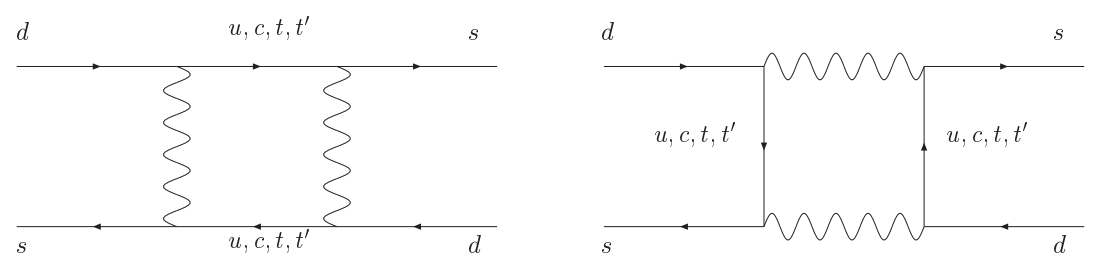

Figure 2. Box diagrams contributing to $K^{0}-\bar{K}^{0}$ mixing.

there are two extra phases in the game. Moreover, even if the extra phases are small, the mixing with the $t^{\prime}$ will change the phase structure of the CKM matrix and new CP violating effects may arise. In the following we will focus on the two most constraining cases: $\Delta F=2$ mixings in the Kaon and B meson sectors.

\subsection{Kaon sector: $K^{0}-\bar{K}^{0}$ mixing}

The $K^{0}-\bar{K}^{0}$ mixing can be calculated in our model with extra non-standard doublet by a simple generalisation of the SM formulas, by including the effect of the $t^{\prime}$ quark as shown in figure 2. In fact, loops involving the flavour violating couplings of the $Z^{0}$ are irrelevant because they only involve up-type quarks. The general formalism to describe such mixing is presented in appendix B: here we will limit ourselves to report the most relevant results. In the non-SM doublet case, the non-diagonal mass matrix element $M_{12}$ between $K^{0}$ and $\bar{K}^{0}$ mesons can be written as

$$
M_{12}=\frac{1}{3} f_{K}^{2} B_{K} m_{K} \frac{G_{F}}{\sqrt{2}} \frac{\alpha}{4 \pi \sin ^{2} \theta_{W}} \sum_{i, j=c, t, t^{\prime}} \eta_{i j} \xi^{i} \xi^{j} E\left(x_{i}, x_{j}\right),
$$

where the Inami-Lim functions $E$ [34] of the mass ratios $x_{i}=m_{i}^{2} / m_{W}^{2}$ are defined in the appendix, and the coefficients $\xi^{i}$ contain the CKM mixings generalised to include $t^{\prime}$ : $\xi^{i}=V_{C K M}^{i s} V_{C K M}^{*, i d}$, with $i=u, c, t, t^{\prime}$. The factors $\eta_{i j}$ encode the QCD loop corrections to 
the electroweak loop diagrams. The condition $V_{C K M}^{\dagger} \cdot V_{C K M}=1$ in eq. (2.27) implies the quadrangle condition $\xi^{u}+\xi^{c}+\xi^{t}+\xi^{t^{\prime}}=0$, therefore one can eliminate the contribution of the up quark loop as in the SM calculation. The functions $E$ are strongly dependent on the value of the masses, and in particular they grow with the mass of the fermions in the loop: to quantify this statement, we list the values of the relevant functions for $m_{t^{\prime}}=350,500,1000 \mathrm{GeV}$ :

$$
\begin{array}{lll}
E\left(x_{c}, x_{c}\right)=2.5 \times 10^{-4}, & E\left(x_{c}, x_{t}\right)=2.2 \times 10^{-3}, & E\left(x_{c}, x_{t^{\prime}}\right)=(2.4,2.5,2.6) \times 10^{-3}, \\
E\left(x_{t}, x_{t}\right)=2.5, & E\left(x_{t}, x_{t^{\prime}}\right)=4.0,4.8,6.4, & E\left(x_{t^{\prime}}, x_{t^{\prime}}\right)=7.5,13,44,
\end{array}
$$

while the function depending on $x_{u}$ are negligible. Here we are interested in two observables: the mass difference $\Delta m_{K}$ between the two mass eigenstates and the CP violating parameter $\epsilon_{K}$, given by

$$
\begin{aligned}
\Delta m_{K} \equiv m_{K_{L}}-m_{K_{S}} & =2 \operatorname{Re} M_{12} \simeq 2\left|M_{12}\right|, \\
\epsilon_{K} & \simeq \frac{e^{i \pi / 4}}{\sqrt{2} \Delta m_{K}} \operatorname{Im} M_{12} .
\end{aligned}
$$

They are to be compared with the experimental results [13]:

$$
\left.\Delta m_{K}\right|_{\exp }=(3.483 \pm 0.006) \times 10^{-15} \mathrm{GeV}, \quad \text { and }\left|\epsilon_{K}\right|_{\exp }=(2.233 \pm 0.015) \times 10^{-3} .
$$

The real part of $M_{12}$, that is related to $\Delta m_{K}$, is dominated by the charm contribution: in fact, the suppression from the function $E$ is compensated by the suppression of the CKM mixing for top and $t^{\prime}$, therefore

$$
\Delta m_{K} \sim 1.75791 \times 10^{-10}\left|\left(V_{c s} V_{c d}^{*}\right)^{2} E\left(x_{c}, x_{c}\right)\right| \sim 2.1 \times 10^{-15} \mathrm{GeV} .
$$

The corrections to $\xi^{c}$ from the new mixing are very small, therefore no significant modification occurs. The situation is rather different for $\epsilon_{K}$. In this case, in the SM the imaginary part of $\xi^{c}$ is of the same order as the imaginary part of $\xi^{t}$, therefore $\epsilon_{K}$ is dominated by the top contribution. Effects of the new physics enter either via modifications of the top couplings and via the new phases, so we can expect large modifications in this case. In addition to $\xi^{i}$, the proportion of the short and long distance contributions in measured values are also important. Compared with the short distance contributions, in the real part of $M_{12}$ the long distance contributions are sizable effects, but in the imaginary part of $M_{12}$ they are negligible. In other words, $\epsilon_{K}$ is more sensitive to the new physics effects than the mass difference $\Delta m_{K}$. The non-diagonal mass matrix element $M_{12}$ of top- and $t^{\prime}$-mediated box diagrams in the vector-like model can be approximately written as

$$
\begin{aligned}
\frac{M_{12}}{C_{K}}= & \left(V_{t s} V_{t d}^{*}\right)^{2}\left\{\tilde{E}\left(x_{t}, x_{t}\right)+2 \frac{m_{t}^{2} \sin ^{2} \theta_{R}}{m_{X}^{2}}\left(\tilde{E}\left(x_{t}, x_{t^{\prime}}\right)-\tilde{E}\left(x_{t}, x_{t}\right)\right)+\frac{m_{t}^{4} \sin ^{4} \theta_{R}}{m_{X}^{4}} \tilde{E}\left(x_{t^{\prime}}, x_{t^{\prime}}\right)\right\} \\
& +\left(V_{t s} V_{t d}^{*}\right) \frac{m_{c}}{m_{t}}\left|V_{R}^{42}\right| \sin \theta_{R} \\
& \times\left\{e^{-i \beta_{2}}\left(V_{t s} V_{c d}^{*}\right) \tilde{E}\left(x_{t}, x_{t}\right)+e^{i \beta_{2}}\left(V_{c s} V_{t d}^{*}\right)\left(\tilde{E}\left(x_{t}, x_{t}\right)+2 \frac{m_{t}^{2}}{m_{t}^{\prime 2}} \tilde{E}\left(x_{t}, x_{t^{\prime}}\right)\right)\right\},
\end{aligned}
$$




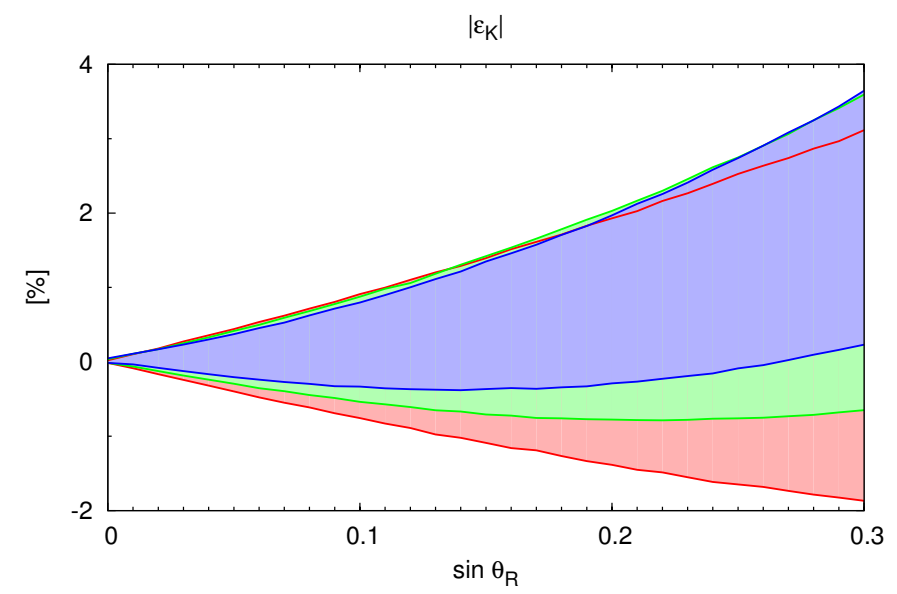

Figure 3. Deviation of $\left|\epsilon_{K}\right|$ in Vector-like model from the SM value is shown as a function of $\sin \theta_{R}$ for three different values of $m_{t^{\prime}}(350 \mathrm{GeV}$ (blue), $500 \mathrm{GeV}$ (green), $1000 \mathrm{GeV}$ (red)). We vary the other parameters within the ranges: $\left|V_{R}^{41}\right| \leq 0.078,\left|V_{R}^{42}\right| \leq 0.2,\left|V_{R}^{41}\right|\left|V_{R}^{42}\right| \leq 3.2 \times 10^{-4}$, $0 \leq \beta_{1}<2 \pi$ and $0 \leq \beta_{2}<2 \pi$.

where $C_{K}=\frac{G_{F}^{2}}{12 \pi^{2}} m_{W}^{2} m_{K} f_{K} B_{K}$ and $\tilde{E}\left(x_{i}, x_{j}\right)=\eta_{i j} E\left(x_{i}, x_{j}\right)$. The contributions from $t^{\prime}$-mediated diagrams with heavy $t^{\prime}$ are suppressed by its mass and these effects become sub-leading terms. The $\beta_{1}$ term is only appear in $\xi^{c}$. Consequently, the result is practically independent of $\beta_{1}$ while it can be modified slightly when varying $\beta_{2}$.

To estimate the range of the effect, we study the correction to $\epsilon_{K}$ numerically. For the SM CKM part $\tilde{V}_{C K M}$, we use the Wolfenstein parameterisation with $\lambda=0.2253, A=0.808$, $\bar{\rho}=0.132$ and $\bar{\eta}=0.341$. In terms of new physics parameters, we take account of tree level bounds which we discussed in previous section. Therefore, we vary new physics parameters within the ranges: $0 \leq \sin \theta_{R} \leq 0.3,\left|V_{R}^{41}\right| \leq 0.078,\left|V_{R}^{42}\right| \leq 0.2,\left|V_{R}^{41}\right|\left|V_{R}^{42}\right| \leq 3.2 \times 10^{-4}$, $0 \leq \beta_{1}<2 \pi$ and $0 \leq \beta_{2}<2 \pi$. Here the upper bound values of $\left|V_{R}^{41}\right|=0.078$ and $\left|V_{R}^{42}\right|=0.2$ correspond to case $\mathrm{A}$ and $\mathrm{B}$ respectively, and the parameter ranges of two new phases $\beta_{1}$ and $\beta_{2}$ in our model are not restricted by the present experimental results. The dependence of $\left|\epsilon_{K}\right|$ in the vector-like model as a function of $\sin \theta_{R}$ is shown in figure 3 for three different values of $m_{t^{\prime}}(350 \mathrm{GeV}$ (blue), $500 \mathrm{GeV}$ (green), $1000 \mathrm{GeV}$ (red)). Deviations are typically at few percent level. The main source of the theoretical uncertainty is the bag parameter $B_{K}$. Note that the effect is always below the theoretical uncertainty in the matrix elements.

\section{2 $B$ meson sector: $B_{d}^{0}-\bar{B}_{d}^{0}$ mixing}

The mixing in the $B$ meson sector can be computed in a similar way as the $K^{0}-\bar{K}^{0}$ mixing. In this case $\Delta M_{B_{q}}=2\left|M_{12}\left(B_{q}\right)\right|, M_{12}\left(B_{q}\right)=\left|M_{12}\left(B_{q}\right)\right| e^{i \Phi_{B_{q}}}$ while the experimental value of $\Delta M_{B_{d}}$ is [13]

$$
\Delta M_{B_{d}}=(3.337 \pm 0.033) \times 10^{-13} \mathrm{GeV} .
$$


In the SM, the real and imaginary parts of $M_{12}\left(B_{d}\right)$ are given by the following forms

$$
\begin{aligned}
\operatorname{Re} M_{12}\left(B_{d}\right) & =C_{B_{d}} \eta_{t t}^{B} E\left(x_{t}, x_{t}\right) A^{2}\left\{(1-\rho)^{2}-\eta^{2}\right\} \lambda^{6}, \\
\operatorname{Im}_{12}\left(B_{d}\right) & =2 C_{B_{d}} \eta_{t t}^{B} E\left(x_{t}, x_{t}\right) A^{2}(1-\rho) \eta \lambda^{6},
\end{aligned}
$$

where $C_{B_{d}}=\frac{G_{F}^{2}}{12 \pi^{2}} m_{B_{d}} f_{B_{d}}^{2} B_{d} m_{W}^{2}$. The leading contribution of $M_{12}\left(B_{d}\right)$ is the top-mediated box diagrams and $\operatorname{Re} M_{12}\left(B_{d}\right)$ is of the same order of magnitude as $\operatorname{Im} M_{12}\left(B_{d}\right)$. To check the leading contribution of $M_{12}\left(B_{d}\right)$ in the vector-like model, we expand $\xi_{d}^{i}$ in a power of $1 / m_{X}^{2}$ :

$$
\begin{aligned}
\frac{M_{12}\left(B_{d}\right)}{C_{B_{d}}}= & \left(V_{t b} V_{t d}^{*}\right)^{2}\left\{\tilde{E}\left(x_{t}, x_{t}\right)+2 \frac{m_{t}^{2} \sin ^{2} \theta_{R}}{m_{X}^{2}}\left(\tilde{E}\left(x_{t}, x_{t^{\prime}}\right)-\tilde{E}\left(x_{t}, x_{t}\right)\right)+\frac{m_{t}^{4} \sin ^{4} \theta_{R}}{m_{X}^{4}} \tilde{E}\left(x_{t^{\prime}}, x_{t^{\prime}}\right)\right\} \\
& -2 e^{+i \beta_{2}}\left(V_{t b} V_{t d}^{*}\right)\left(V_{t b} V_{c d}^{*}\right) \frac{m_{c}}{m_{t}}\left|V_{R}^{42}\right| \sin \theta_{R} \\
& \times\left\{\tilde{E}\left(x_{t}, x_{t}\right)-\frac{m_{t}^{2}}{m_{t}^{\prime 2}} \tilde{E}\left(x_{t}, x_{t^{\prime}}\right)-\frac{m_{t}^{4} \sin ^{2} \theta_{R}}{m_{t}^{\prime 2} m_{X}^{2}} \tilde{E}\left(x_{t^{\prime}}, x_{t^{\prime}}\right)\right\}
\end{aligned}
$$

where $\tilde{E}\left(x_{i}, x_{j}\right)=\eta_{i j}^{B} E\left(x_{i}, x_{j}\right)$. The function $E\left(x_{t^{\prime}}, x_{t^{\prime}}\right) \simeq x_{t^{\prime}}$ grows with the $t^{\prime}$ mass, while the coefficient $\left(\xi_{d}^{t^{\prime}}\right)^{2}$ is suppressed by $1 / m_{t^{\prime}}^{4}$. Consequently, deviations which comes from this effect are no more than $m_{t}^{2} / m_{X}^{2}$. Similarly, the top- $t^{\prime}$-mediated box diagrams are also suppressed by a power of $m_{t}^{2} / m_{t^{\prime}}^{2} \sim m_{t}^{2} / m_{X}^{2}$. Therefore these new physics effects are sub-leading terms in allowed parameter space. It is important to check the new physics effect which is caused by the modification of the CKM matrix. The contributions from the top-mediated diagrams are written as follows:

$$
\begin{aligned}
\operatorname{Re}_{12}\left(B_{d}\right)= & C_{B_{d}} \eta_{t t}^{B} E\left(x_{t}, x_{t}\right)\left[\left(1-2 \frac{m_{t}^{2} \sin ^{2} \theta_{R}}{m_{X}^{2}}\right) A^{2}\left\{(1-\rho)^{2}-\eta^{2}\right\} \lambda^{6}\right. \\
& \left.+2 \frac{m_{c}}{m_{t}}\left|V_{R}^{42}\right| \sin \theta_{R} A\left\{(1-\rho) \cos \beta_{2}+\eta \sin \beta_{2}\right\} \lambda^{4}\right], \\
\operatorname{Im}_{12}\left(B_{d}\right)= & 2 C_{B_{d}} \eta_{t t}^{B} E\left(x_{t}, x_{t}\right)\left[\left(1-2 \frac{m_{t}^{2} \sin ^{2} \theta_{R}}{m_{X}^{2}}\right) A^{2}(1-\rho) \eta \lambda^{6}\right. \\
& \left.-\frac{m_{c}}{m_{t}}\left|V_{R}^{42}\right| \sin \theta_{R} A\left\{(1-\rho) \sin \beta_{2}-\eta \cos \beta_{2}\right\} \lambda^{4}\right] .
\end{aligned}
$$

The second term is proportional to $\lambda^{7}$ due to $\frac{m_{c}}{m_{t}} \sim \mathcal{O}\left(\lambda^{3}\right)$. Therefore, in the $B_{d}^{0}-\bar{B}_{d}^{0}$ mixing, we find that new physics contributions are smaller than the SM ones.

In the $B_{d}$ system, numerical results are analogous to $K^{0}-\bar{K}^{0}$ mixing as typical difference from the standard model value of $\Delta M_{B_{d}}$ is in the few to $10 \%$ range. These values are below the theoretical uncertainty in the matrix elements. In our numerical calculations, we set $\eta_{t t}^{B}=\eta_{t t^{\prime}}^{B}=\eta_{t^{\prime} t^{\prime}}^{B}$. Although this is not accurate, the discrepancy between our numerical results and the results including NLO QCD corrections is small because the top mediated box diagrams are the leading contributions in our model. We plot the real and imaginary parts of $M_{12}\left(B_{d}\right)$ for $m_{t^{\prime}}=350,500,1000 \mathrm{GeV}$ in figure 4 . All the effects are typically small deviations with respect to the SM in the $B_{d}$ system. 

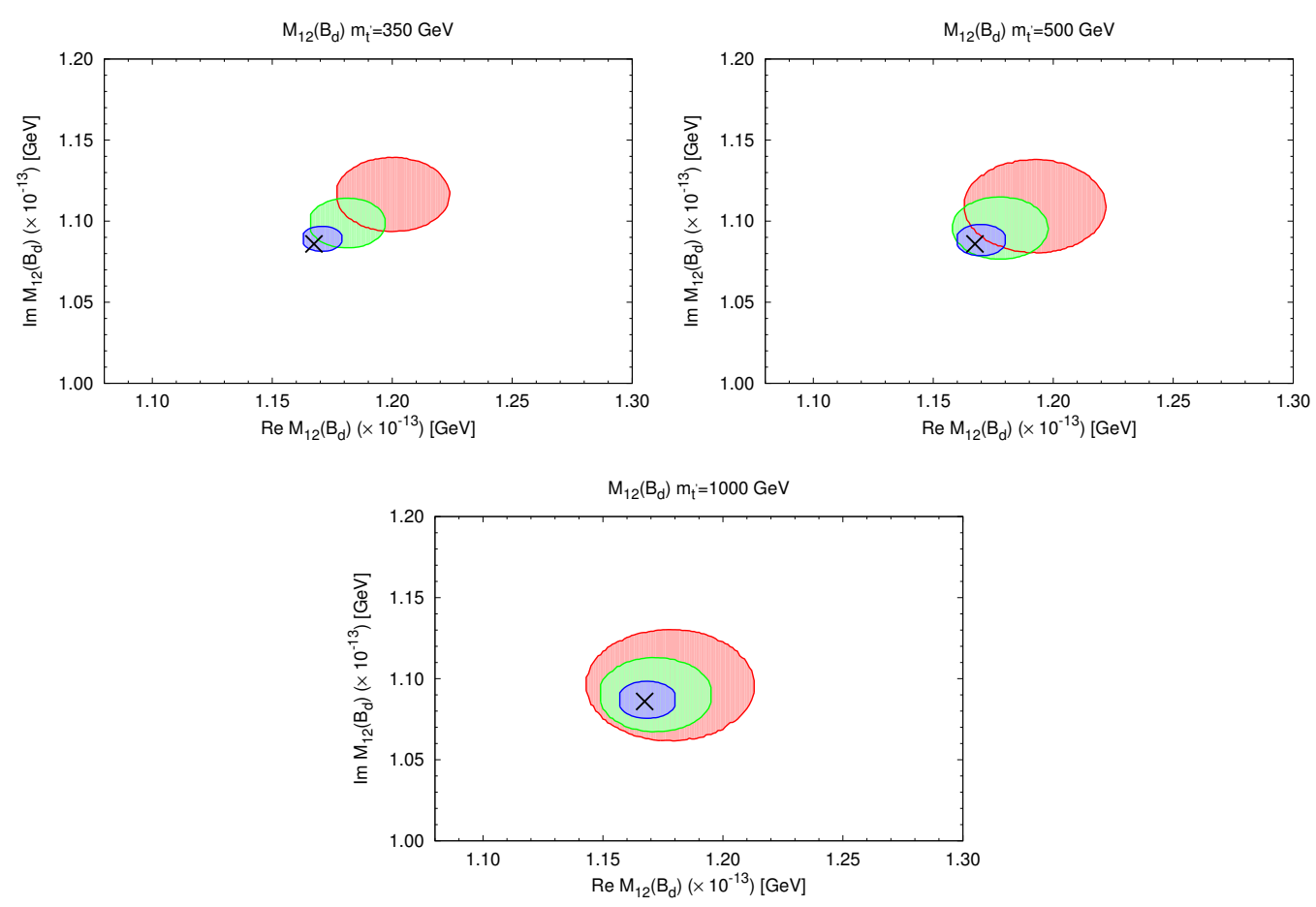

Figure 4. Real and imaginary parts of $M_{12}\left(B_{d}\right)$ for $m_{t^{\prime}}=350,500,1000 \mathrm{GeV}$. The Vector-like model is shown for three different values of $\sin \theta_{R}$ (0.1 (blue), 0.2 (green), 0.3 (red)). The SM value is shown with a black cross. We set that $\left|V_{R}^{41}\right| \leq 0.078,\left|V_{R}^{42}\right| \leq 0.2,\left|V_{R}^{41}\right|\left|V_{R}^{42}\right| \leq 3.2 \times 10^{-4}$, $0 \leq \beta_{1}<2 \pi$ and $0 \leq \beta_{2}<2 \pi$.

\section{3 $B$ meson sector: $B_{s}^{0}-\bar{B}_{s}^{0}$ mixing}

The experimental value of $\Delta M_{B_{s}}$ is

$$
\Delta M_{B_{s}}=(1.170 \pm 0.008) \times 10^{-11} \mathrm{GeV} .
$$

The real and imaginary parts of $M_{12}\left(B_{s}\right)$ in the SM can be written as

$$
\begin{aligned}
\operatorname{Re} M_{12}\left(B_{s}\right) & =C_{B_{s}} \eta_{t t}^{B} E\left(x_{t}, x_{t}\right) A^{2} \lambda^{4}, \\
\operatorname{Im}_{12} M_{12}\left(B_{s}\right) & =-2 C_{B_{s}} \eta_{t t}^{B} E\left(x_{t}, x_{t}\right) A^{2} \eta \lambda^{6},
\end{aligned}
$$

where $C_{B_{s}}=\frac{G_{F}^{2}}{12 \pi^{2}} m_{B_{s}} f_{B_{s}}^{2} B_{s} m_{W}^{2}$. In comparison with the real part $R e M_{12}\left(B_{s}\right)$, the imaginary part $\operatorname{ImM}_{12}\left(B_{s}\right)$ is suppressed by a power of $\lambda^{2}$. This is a remarkable feature of $B_{s}^{0}-\bar{B}_{s}^{0}$ mixing. Even though the new physics effects to $\operatorname{Re} M_{12}\left(B_{s}\right)$ are small, there is some possibility of that $\operatorname{Im} M_{12}\left(B_{s}\right)$ is largely shifted by the new CP phases. In the 
vector-like model, $M_{12}\left(B_{s}\right)$ can be written as

$$
\begin{aligned}
\frac{M_{12}\left(B_{s}\right)}{C_{B_{s}}}= & \left(V_{t b} V_{t s}^{*}\right)^{2}\left\{\tilde{E}\left(x_{t}, x_{t}\right)+2 \frac{m_{t}^{2} \sin ^{2} \theta_{R}}{m_{X}^{2}}\left(\tilde{E}\left(x_{t}, x_{t^{\prime}}\right)-\tilde{E}\left(x_{t}, x_{t}\right)\right)+\frac{m_{t}^{4} \sin ^{4} \theta_{R}}{m_{X}^{4}} \tilde{E}\left(x_{t^{\prime}}, x_{t^{\prime}}\right)\right\} \\
& -2 e^{+i \beta_{2}}\left(V_{t b} V_{t s}^{*}\right)\left(V_{t b} V_{c s}^{*}\right) \frac{m_{c}}{m_{t}}\left|V_{R}^{42}\right| \sin \theta_{R} \\
& \times\left\{\tilde{E}\left(x_{t}, x_{t}\right)-\frac{m_{t}^{2}}{m_{t}^{\prime 2}} \tilde{E}\left(x_{t}, x_{t^{\prime}}\right)-\frac{m_{t}^{4} \sin ^{2} \theta_{R}}{m_{t}^{\prime 2} m_{X}^{2}} \tilde{E}\left(x_{t^{\prime}}, x_{t^{\prime}}\right)\right\} .
\end{aligned}
$$

It is found that the top-mediated box diagrams become leading contribution in the $B_{s}^{0}-\bar{B}_{s}^{0}$ mixing. The real and imaginary part of $M_{12}\left(B_{s}\right)$ due to the top-mediated box diagrams can be expressed analogously to the $M_{12}\left(B_{d}\right)$ as

$$
\begin{aligned}
\operatorname{Re} M_{12}\left(B_{s}\right)= & C_{B_{s}} \eta_{t t}^{B} E\left(x_{t}, x_{t}\right) \\
& \times\left[\left(1-2 \frac{m_{t}^{2} \sin ^{2} \theta_{R}}{m_{X}^{2}}\right) A^{2} \lambda^{4}+\frac{m_{c}}{m_{t}}\left|V_{R}^{42}\right| \sin \theta_{R} \cos \beta_{2} A \lambda^{2}\right], \\
\operatorname{ImM}_{12}\left(B_{s}\right)= & -2 C_{B_{s}} \eta_{t t}^{B} E\left(x_{t}, x_{t}\right) \\
& \times\left[\left(1-2 \frac{m_{t}^{2} \sin ^{2} \theta_{R}}{m_{X}^{2}}\right) A^{2} \eta \lambda^{6}+\frac{m_{c}}{m_{t}}\left|V_{R}^{42}\right| \sin \theta_{R} \sin \beta_{2} A \lambda^{2}\right] .
\end{aligned}
$$

In the imaginary part of $M_{12}\left(B_{s}\right)$, this shows that the new physics contributions are sizable effects in comparison with the SM prediction for $\left|V_{R}^{42}\right|=0.2$ (case B), $\sin \theta_{R}=0.3$ and $\beta_{2}= \pm \frac{\pi}{2}$. It is found that the $\left|V_{R}^{14}\right|$ and $\beta_{1}$ dependences are negligible. This large effect completely vanish when $\beta_{2}=0, \pi$. In case $\mathrm{A}$, the correction to $\operatorname{Im} M_{12}\left(B_{s}\right)$ cannot be large due to the small value of $\left|V_{R}^{42}\right|\left(\left|V_{R}^{42}\right| \leq 3.2 \times 10^{-4} /\left|V_{R}^{41}\right|\right.$ with $\left.\left|V_{R}^{41}\right|=0.078\right)$. It is also found that the $t^{\prime}$ mass dependence of the imaginary part of $M_{12}\left(B_{s}\right)$ is quite small, because the second term in eq. (4.17) is the leading new physics contribution and does not depend on the $t^{\prime}$ mass. In the real part of $M_{12}\left(B_{s}\right)$, new physics effect is proportional to $\mathcal{O}\left(\lambda^{5}\right)$. Therefore this effect is at least smaller than the SM value by a factor of $\lambda$. The numerical results of real and imaginary parts of $M_{12}\left(B_{s}\right)$ for $m_{t^{\prime}}=350,500,1000 \mathrm{GeV}$ are shown in figure 5. It is found that the deviations of $\operatorname{Re} M_{12}\left(B_{s}\right)$ are less than $10 \%$, while the deviations of $\operatorname{Im} M_{12}\left(B_{s}\right)$ are about $\pm 100 \%$ for $\sin \theta_{R}=0.3$. These numerical results agree well with the analytical results in eq.s (4.16) and (4.17).

In the $B_{s}$ system deviations of $\Delta M_{B_{s}}$ and the phase $\Phi_{s}$ with respect to the SM are at few percent level for $\beta_{1}=\beta_{2}=0$. When $\beta_{1}=\beta_{2}=0$ the phase $\Phi_{s}$ in the vector-like model is very close to zero in both cases A and B. However the situation can be drastically different when $\beta_{2}$ is non-zero, see figure 6 . Here we define the deviation of the phase $\Phi_{s}$ in the vector-like model from the SM value as $\Delta \Phi_{s}=\left(\Phi_{s}^{\mathrm{Vec}}-\Phi_{s}^{\mathrm{SM}}\right) / \Phi_{s}^{\mathrm{SM}}$. For $\beta_{2}=\frac{\pi}{2}$ $\left(-\frac{\pi}{2}\right)$, it becomes maximal (minimum) value in case B. The deviation of the phase $\Delta \Phi_{s}$ can be enhanced by $+40(+60) \%$ for $m_{t^{\prime}}=350(1000) \mathrm{GeV}$ and $\sin \theta_{R}=0.3$ due to the large deviation of $\operatorname{Im} M_{12}\left(B_{s}\right)$. For $\beta_{2}=-\frac{\pi}{2}$, it becomes negative deviation $-90(-150) \%$. A large phase is naturally allowed within our vector-like model with a non-standard doublet of quarks. This feature is also present in other models beyond the SM. A detailed study of this feature, in particular in relation with the dimuon asymmetry in $B_{s}^{0}-\bar{B}_{s}^{0}$ mixing will be described in a separate paper. 

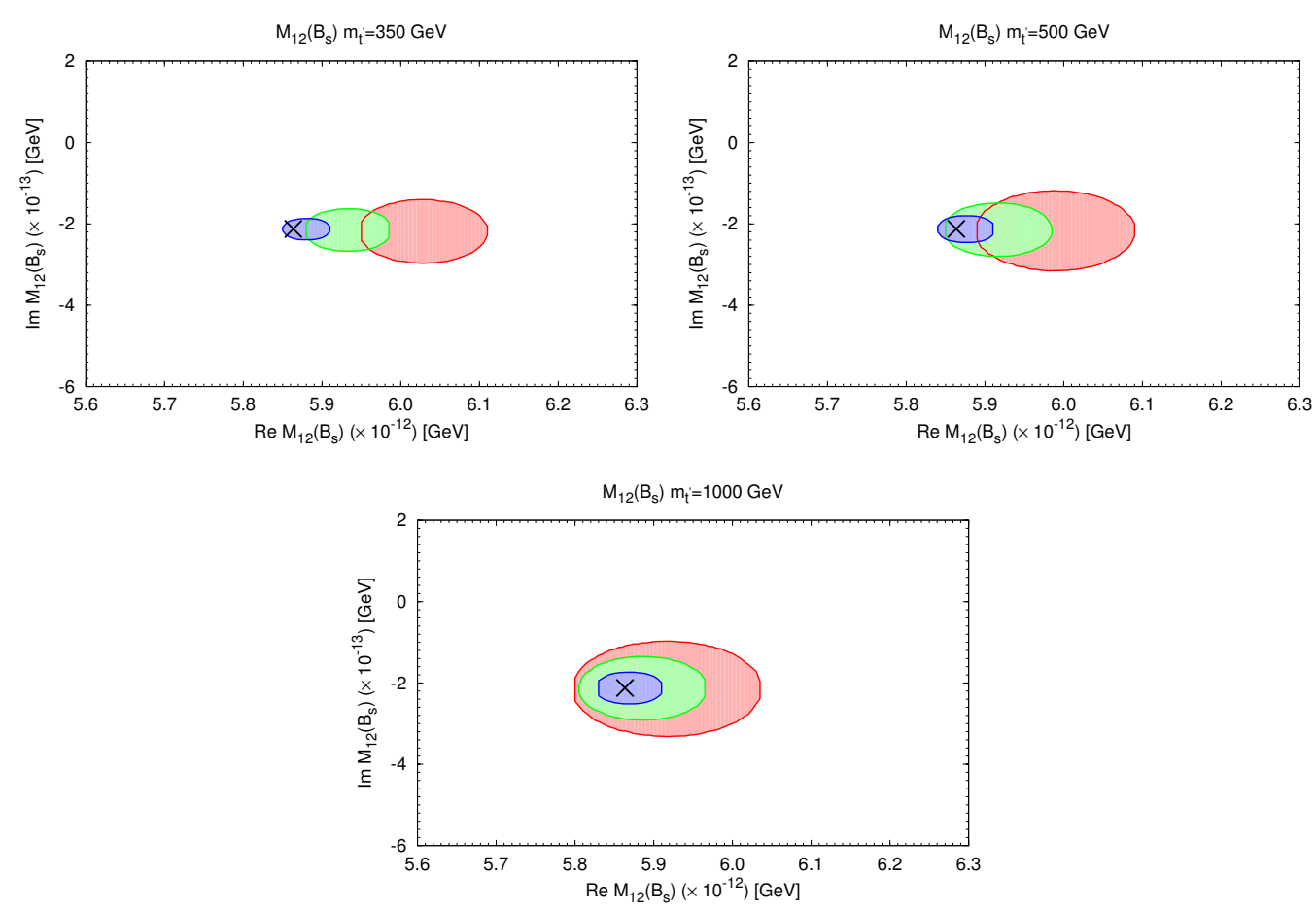

Figure 5. Real and imaginary parts of $M_{12}\left(B_{s}\right)$ for $m_{t^{\prime}}=350,500,1000 \mathrm{GeV}$. The Vector-like model is shown for three different values of $\sin \theta_{R}$ (0.1 (blue), 0.2 (green), 0.3 (red)). The SM value is shown with a black cross. We vary the other parameters within the ranges $\left|V_{R}^{14}\right| \leq 0.078$, $\left|V_{R}^{24}\right| \leq 0.5,\left|V_{R}^{14}\right|\left|V_{R}^{24}\right| \leq 3.2 \times 10^{-4}, 0 \leq \beta_{1}<2 \pi$ and $0 \leq \beta_{2}<2 \pi$.

We also plot the ratio $\left(\frac{\Delta M_{B_{s}}}{\Delta M_{B_{d}}}\right)$ in figure 7 , which is less affected by theoretical uncertainties. However the deviation with respect to the standard model is small (at most 1\%).

\subsection{Coupling of the Higgs to gluons and photons}

An important effect of new states that couple to the Higgs is to affect the loop-induced couplings to gluons and photons. Phenomenologically, this is of crucial importance at the LHC, because the main Higgs production mechanism is gluon fusion and, for low masses, the golden discovery channel is in two photons. Even though the new fermion could be heavy, the effect on the loop may be significant.

We can easily calculate the effect on the loop by using the parameterisation proposed in [35]: if we neglect the contribution of the light fermions, the only contributions will come from the couplings of the $t^{\prime}$ and from modifications of the top couplings. The two parameters can be written as:

$$
\kappa_{g g}=\kappa_{\gamma \gamma}=\frac{v}{m_{t}} h_{h t \bar{t}}+\frac{v}{m_{t^{\prime}}} h_{h t^{\prime} \bar{t}^{\prime}}-1
$$

where $h_{h f \bar{f}}$ is the coupling of the Higgs with the fermion $f$. From eq. (2.32), we have

$$
h_{h t \bar{t}}=\frac{m_{t}}{v}-\frac{M}{v} V_{L}^{*, 43} V_{R}^{43}, \quad \text { and } \quad h_{h t^{\prime} \bar{t}^{\prime}}=\frac{m_{t^{\prime}}}{v}-\frac{M}{v} V_{L}^{*, 44} V_{R}^{44} .
$$




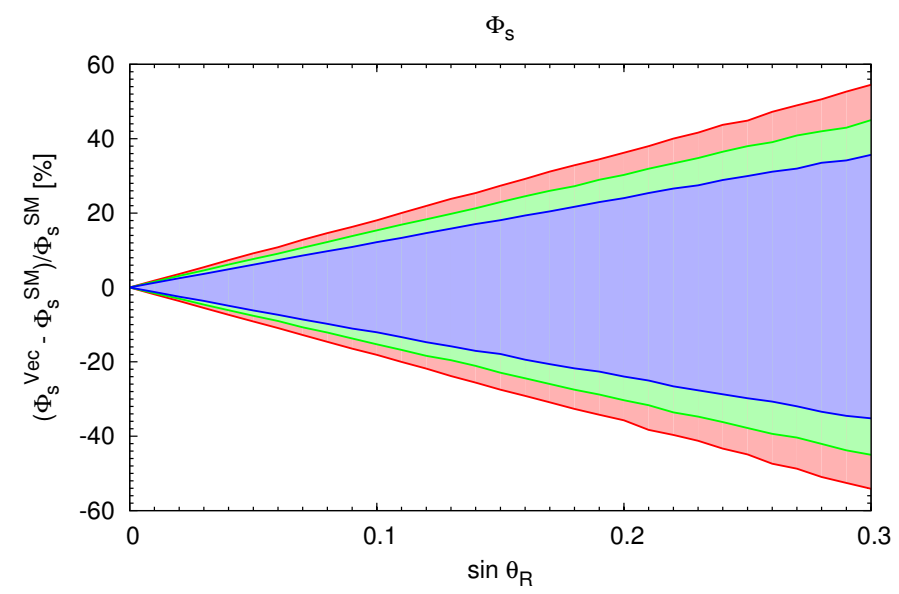

Figure 6. Deviation of $\Phi_{s}$ in Vector-like model from the SM value is shown as a function of $\sin \theta_{R}$ for three different values of $m_{t^{\prime}}(350 \mathrm{GeV}$ (blue), $500 \mathrm{GeV}$ (green), $1000 \mathrm{GeV}$ (red)). We vary the other parameters within the ranges $\left|V_{R}^{14}\right| \leq 0.078,\left|V_{R}^{24}\right| \leq 0.5,\left|V_{R}^{14}\right|\left|V_{R}^{24}\right| \leq 3.2 \times 10^{-4}, 0 \leq \beta_{1}<2 \pi$ and $0 \leq \beta_{2}<2 \pi$.

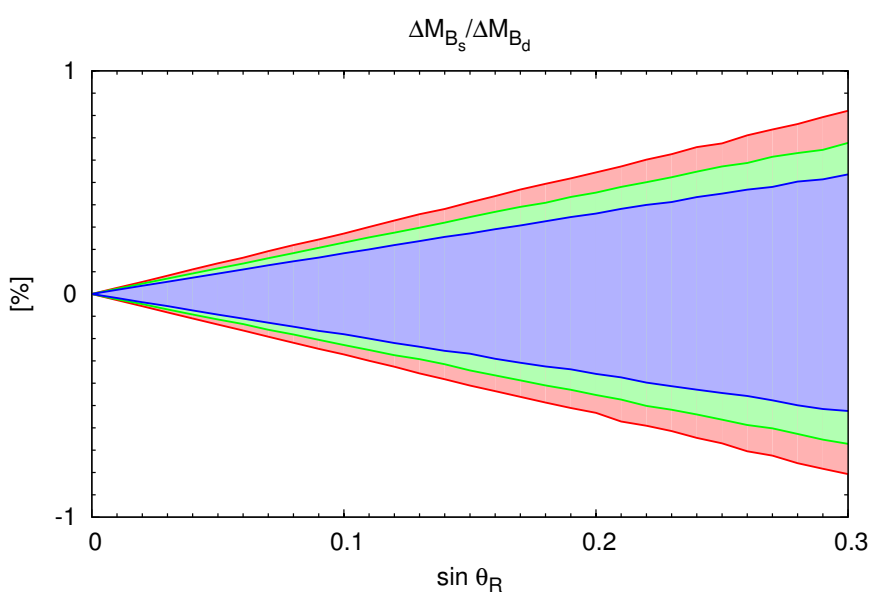

Figure 7. Deviation of $\left(\frac{\Delta M_{B_{s}}}{\Delta M_{B_{d}}}\right)$ in Vector-like model from the SM value is shown as a function of $\sin \theta_{R}$ for three different values of $m_{t^{\prime}}(350 \mathrm{GeV}$ (blue), $500 \mathrm{GeV}$ (green), $1000 \mathrm{GeV}$ (red)). We vary the other parameters within the ranges $\left|V_{R}^{14}\right| \leq 0.078,\left|V_{R}^{24}\right| \leq 0.5,\left|V_{R}^{14}\right|\left|V_{R}^{24}\right| \leq 3.2 \times 10^{-4}$, $0 \leq \beta_{1}<2 \pi$ and $0 \leq \beta_{2}<2 \pi$.

Therefore:

$$
\kappa_{g g}=\kappa_{\gamma \gamma}=1-\frac{M}{m_{t}} V_{L}^{*, 34} V_{R}^{34}-\frac{M}{m_{t^{\prime}}} V_{L}^{*, 44} V_{R}^{44}=\frac{\left(\left|x_{1}\right|^{2}+\left|x_{2}\right|^{2}\right)\left(2-\sin ^{2} \theta_{R}\right)}{2 m_{X}^{2}} .
$$

It is interesting to notice that this result is rather small, being proportional to the mixing with the light generations, and does not depend significantly on the new coupling of the top $x_{3}$, as we may have naively expected. The reason for this is the following: if we take 


\begin{tabular}{|c|c|c|c|c|c|}
\hline & $m_{t^{\prime}}(\mathrm{GeV})$ & $\sin \theta_{R}$ & $V_{R}^{14}$ & $V_{R}^{24}$ & $m_{H}(\mathrm{GeV})$ \\
value & 350,500 & 0.3 & 0.077 & 0.0025 & 120,1000 \\
\hline
\end{tabular}

Table 3. Benchmark point values of the parameters. The ones with two values will be specified in each case.

the inverse of eq. (2.13), we obtain the relation

$$
M_{u}^{-1}=V_{R} \cdot\left(\begin{array}{cccc}
\frac{1}{m_{u}} & & & \\
& \frac{1}{m_{c}} & & \\
& & \frac{1}{m_{t}} & \\
& & & \frac{1}{m_{t^{\prime}}}
\end{array}\right) \cdot V_{L}^{\dagger} .
$$

The component 44 of this matrix is

$$
\left(M_{u}^{-1}\right)_{44}=\frac{1}{M}=\sum_{i=1}^{4} \frac{1}{m_{i}} V_{R}^{4 i} V_{L}^{*, 4 i} .
$$

In the case of mixing only between top and new fermion, $x_{1}=x_{2}=0$, the relation reduces to

$$
\sum_{i=3,4} \frac{M}{m_{i}} V_{R}^{4 i} V_{L}^{*, 4 i}=1 .
$$

Finally, in the 2 fermion case, $V_{L, R}^{43}=-V_{L, R}^{34}=-\sin \theta_{L, R}$, therefore this relation implies

$$
\frac{M}{m_{t}} V_{L}^{*, 34} V_{R}^{34}+\frac{M}{m_{t^{\prime}}} V_{L}^{*, 44} V_{R}^{44}=1
$$

This relation implies that $\kappa_{g g}=\kappa_{\gamma \gamma}$ vanish in the 2 fermion mixing case. From eq. (4.19), we can extract an upper bound on the $\kappa$ parameters. Using the relation $\left|V_{R}^{41}\right|^{2}+\left|V_{R}^{42}\right|^{2}=$ $\frac{1}{m_{X} 2}\left(\left|x_{1}\right|^{2}+\left|x_{2}\right|^{2}\right)$ and the bound from $t \rightarrow Z j$ decays in eq. (3.22), we obtain

$$
k a p p a_{\gamma}=\kappa_{g g}=\left(\left|V_{R}^{41}\right|^{2}+\left|V_{R}^{42}\right|^{2}\right) \lesssim 0.04 .
$$

The upper bound is saturated by the maximal value of $V_{R}^{42}$ : this shows that loop effects are very small and therefore the Higgs phenomenology is the same as in the standard model.

\section{LHC phenomenology}

In the following we consider the LHC phenomenology for the decay of a heavy vectorlike quark. Few promising decay modes are analysed and their relevance with respect to backgrounds is discussed. In this section we will present our numerical results for the benchmark values of the parameters in table 3 . The value of the mixing $V_{R}^{12}$ in particular is fixed to its maximal value allowed by precision measurements, while $V_{R}^{14}$ maximises the bound from the $D^{0}-\bar{D}^{0}$ mixing. 

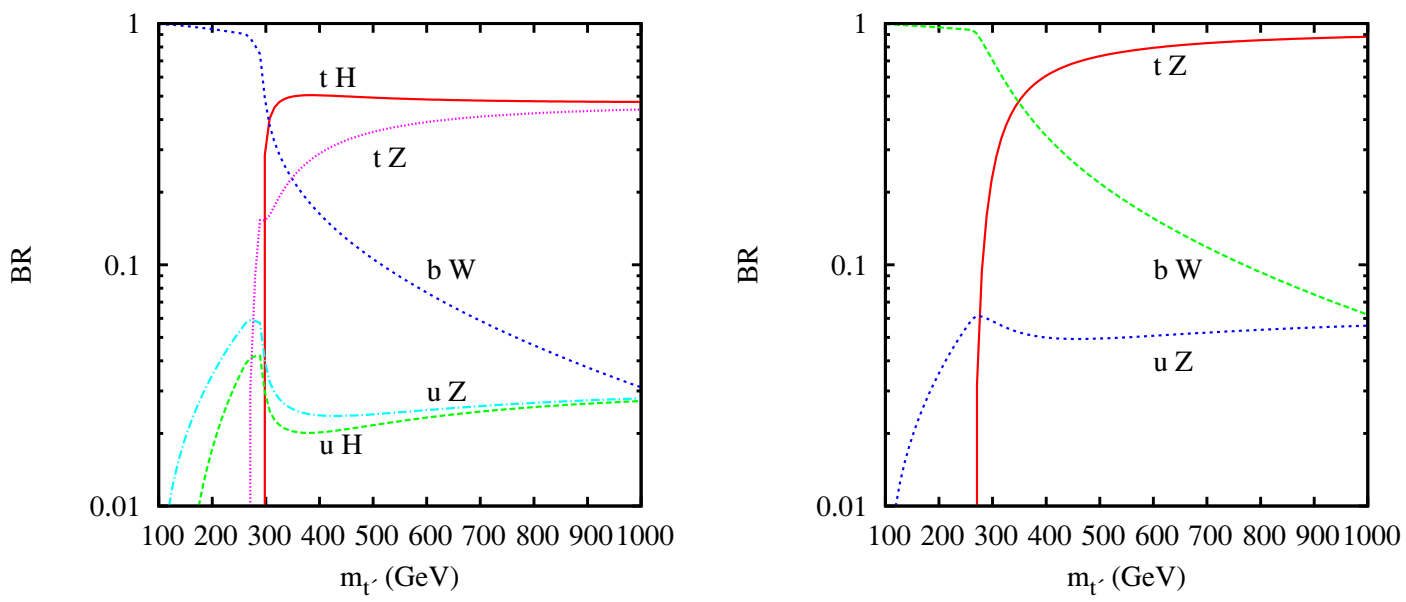

Figure 8. BR of $t^{\prime}$ as function of $m_{t^{\prime}}$ for the benchmark parameters in table 3 . The two panels correspond to a Higgs mass $m_{h}=120 \mathrm{GeV}$ (left panel) and $m_{h}$ decoupled from the spectrum (right panel).

\subsection{Decays}

The branching ratios for the decay of a heavy vector-like quark are quite different from those of a sequential fourth family and therefore this is an important physical characteristics to distinguish the two different situations. The phenomenology is also novel as vector-like quarks can decay at tree level via neutral currents, to a SM fermions plus a $Z / h$, while a fourth generation quark can only decay via the $W$ boson. A detailed and general discussion of the parameter and case dependence is given in appendix B2 of [14]. Here, we will focus on the specific case of the non-standard vector-like doublet containing a new $t^{\prime}$. In figure 8 we show the branching rations for a light Higgs with $m_{h}=120 \mathrm{GeV}$ and for a decoupled Higgs $\left(m_{H}=1000 \mathrm{GeV}\right)$. All the other parameters are fixed to the benchmark values in table 3.

For light $t^{\prime}$ masses, below the $t Z$ and $t h$ thresholds, the decay is dominated by the $W b$ channel, like for a fourth generation. However, after the $t h$ channel opens up, it quickly saturates to a BR of $50 \%$, while $t Z$ more slowly replaces $W b$ for large masses. This shows that the final states produced by the decays of the vector-like $t^{\prime}$ are very different from the ones typical of a fourth generation. The trend is similar in the case of a decoupled Higgs, apart for the absence of the th channel.

\subsection{Single production}

The single production of $t^{\prime}$ takes place via flavour violating interactions of the $Z$ and $W$. The $W$ mediated channels are similar to the ones giving single top, however the mixing angles depend on the mixing matrix in the left-handed sector and they are very suppressed. Therefore, the production is dominated by the exchange, in t- and s-channel, of the $Z$ boson depicted in figure 9 where the grey blob represents the flavour violating coupling. Such process is absent in the case of a fourth generation. The production cross-section is shown on the left panel of figure 10 as a function of $m_{t^{\prime}}$ for the benchmark parameters in table 3 . 


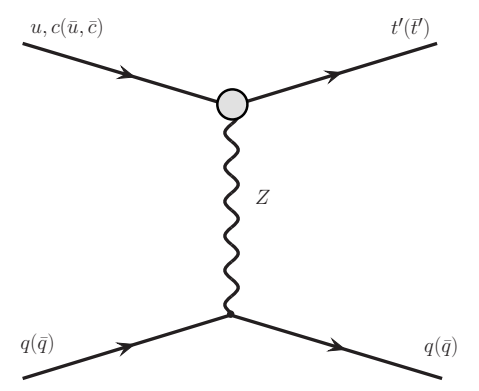

Figure 9. Feynman diagram for the single production of $t^{\prime}$ at LHC.

\begin{tabular}{|c|c|c|c|c|c|c|}
\hline & $\sin \theta_{R}$ & $\left(m_{t^{\prime}}, m_{h}\right)$ & \multicolumn{3}{|c|}{$\sigma$} & \multicolumn{3}{|c|}{ Branching Ratios of $t^{\prime}$} \\
& & $(\mathrm{GeV})$ & $(\mathrm{fb})$ & $t Z$ & $t h$ & $W b$ \\
\hline BP 1 & 0.3 & $(350,120)$ & 691 & 0.23 & 0.51 & 0.23 \\
BP 2 & 0.3 & $(350,1000)$ & 691 & 0.50 & - & 0.49 \\
BP 3 & 0.3 & $(500,120)$ & 254 & 0.36 & 0.50 & 0.10 \\
BP 4 & 0.3 & $(500,1000)$ & 254 & 0.77 & - & 0.23 \\
\hline & & & & $u Z$ & $u h$ & \\
\hline BP 1-A & 0 & $(350,120)$ & 436 & 0.13 & 0.87 & \\
BP 2-A & 0 & $(500,120)$ & 223 & 0.06 & 0.93 & \\
\hline
\end{tabular}

Table 4. Benchmark points, production cross-section $(\sigma)$ and Branching ratios of $t^{\prime}$ for the results of LHC simulations.

The dominant contribution comes from the t-channel exchange of the $Z$ with a valence up quark in the initial state, therefore it is directly proportional to the mixing angle $V_{R}^{41}$. The benchmark point we chose is characterised by a maximal $V_{R}^{41}$, therefore the values of the cross-section in the figure are the maximal values in this model. On the right panel, we also show the pair production cross-section as a function of $m_{t^{\prime}}$ : one important difference is that the single production cross-section decreases more slowly compared to the pair production and, for masses above $450 \mathrm{GeV}$ it dominates at $7 \mathrm{TeV}$. In the following we will study in detail the single production case, which seems more promising at $7 \mathrm{TeV}$, and leave the pair production case for future investigation.

In table 4, we listed the values of the cross-section and branching ratios in a few benchmark points that we will consider more in detail in the following. As the general mixing structure is taken into account, decays to first and second generation quarks are allowed and controlled by the mixing parameters. In the case $\sin \theta_{R}=0$, points 1 -A and 2 -A, the mixing with the top vanishes, therefore the $t^{\prime}$ can only decay into a light quark plus $Z / h$. The decay into a $W$ is suppressed by the light quark masses.

The production cross-sections and branching ratios have been evaluated with CalcHEP [36] with CTEQ61 PDFs and the factorization scale set to be $m_{t^{\prime}}\left(2 m_{t^{\prime}}\right.$ for the pair production).

As shown in figure 8 the dominant decay mode of $t^{\prime}$ are $t^{\prime} \rightarrow$ th (for a light Higgs) and $t^{\prime} \rightarrow t Z$. Therefore the possible signatures of single $t^{\prime}$ production at LHC are: 

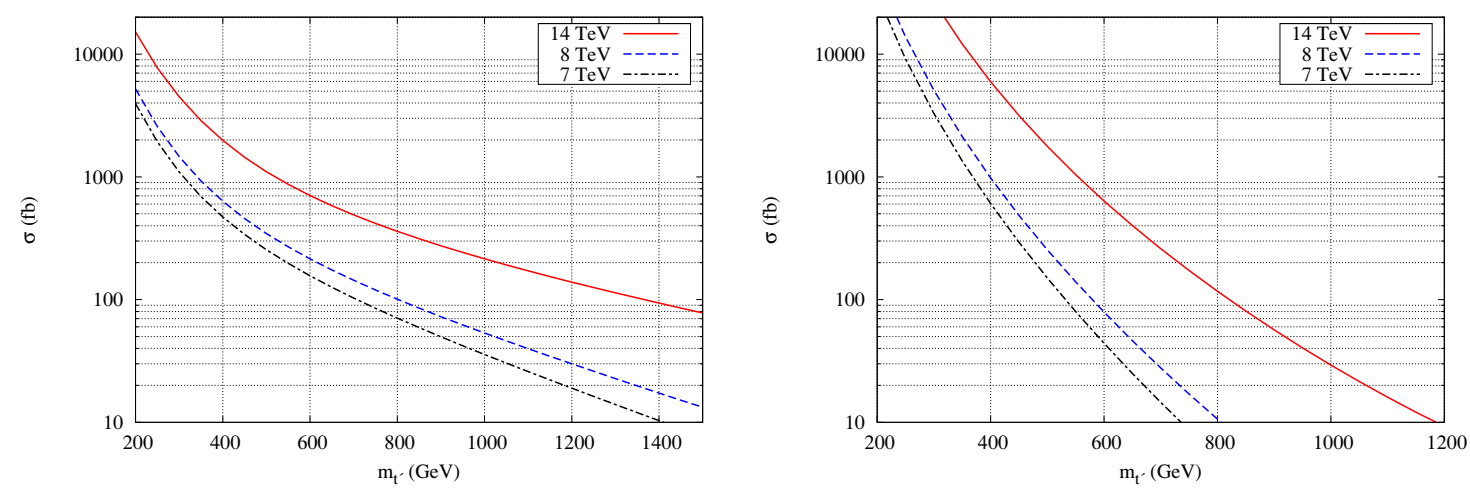

Figure 10. Production cross-section for a single $p p \rightarrow t^{\prime} j$ (left) and a pair $p p \rightarrow t^{\prime} \bar{t}^{\prime}$ (right) of heavy quarks as a function of $m_{t^{\prime}}$ for the benchmark parameters in table 3 . We consider various energy options for the LHC.

1. $p p \rightarrow b j \ell^{ \pm} \mathbb{E}_{T}$ via the decay chains

$$
\begin{aligned}
& t^{\prime} \rightarrow t\left(\rightarrow b \ell^{ \pm} \nu\right) Z(\rightarrow \nu \bar{\nu}) \rightarrow b \ell^{ \pm} E_{T} \\
& t^{\prime} \rightarrow b W(\rightarrow \ell \nu) \rightarrow b \ell^{ \pm} E_{T}
\end{aligned}
$$

2. $p p \rightarrow b \ell^{ \pm} \ell^{\mp} \ell^{ \pm} j E_{T}$ via the decay chain

$$
t^{\prime} \rightarrow t(\rightarrow b \ell \nu) Z\left(\rightarrow \ell^{+} \ell^{-}\right)
$$

3. $p p \rightarrow b \bar{b} b j \ell^{ \pm} E_{T}$ via the decay chain

$$
t^{\prime} \rightarrow t H(\rightarrow b \bar{b}) \rightarrow t(\rightarrow b \ell \nu) b \bar{b} \rightarrow b \bar{b} b \ell E_{T}
$$

4. $p p \rightarrow b \ell^{ \pm} j j j E_{T}$ via the decay chain

$$
t^{\prime} \rightarrow t(\rightarrow b \ell \nu) Z(\rightarrow j j)
$$

In addition to these channels we have also considered a very interesting possibility when $\sin \theta_{R}=0$. This corresponds to no-mixing between $t$ and $t^{\prime}$ and hence the decay channel $t^{\prime} \rightarrow t Z / h$ is closed and the dominant decay modes of $t^{\prime}$ are $t^{\prime} \rightarrow u Z / h$.

In the following sections, we will discuss each of these signatures separately.

\subsubsection{Framework of event generation and analysis}

The setup used for signal and background event generation is the following:

- Signal events: the partonic level signal events were generated by CalcHEP 2.5.6 [36]. The partonic level events were then interfaced with PYTHIA 6.4.21 [37] via the LHE (Les Houches Event) interface in order to include ISR/FSR and hadronization. 
- Background events: the $t \bar{t}$ background was simulated using PYTHIA 6.4.21. The single top and $Z+$ jets backgrounds were generated using ALPGEN 2.14 [38]. The backgrounds for the signature $b j \ell^{ \pm} \ell^{\mp} \ell^{ \pm} E_{T}$ were generated using Madgraph [39].

We have used a modified version of ATLFAST [40] to reconstruct the jets and identify the isolated particles. Finally the events were analyzed within ROOT framework. For our analysis we have considered LHC with centre of mass energy of $7 \mathrm{TeV}$ and an integrated luminosity of $10 \mathrm{fb}^{-1}$. In our analysis leptons are intended to be only electrons and muons $(\ell=e, \mu)$.

\subsubsection{Signature $p p \rightarrow t^{\prime}(\rightarrow t Z) j \rightarrow b j \ell^{ \pm} E_{T}$}

This signature originates from two independent decay chains:

$$
\begin{aligned}
& t^{\prime} \rightarrow t\left(\rightarrow b \ell^{ \pm} \nu\right) Z(\rightarrow \nu \bar{\nu}) \rightarrow b \ell^{ \pm} E_{T} \\
& t^{\prime} \rightarrow b W(\rightarrow \ell \nu) \rightarrow b \ell^{ \pm} E_{T}
\end{aligned}
$$

The effective cross-section of this process is

$$
\begin{aligned}
\sigma^{\mathrm{eff}} & =\sigma \times\left[B R\left(t^{\prime} \rightarrow t Z\right) \times B R(Z \rightarrow \nu \bar{\nu}) \times B R(t \rightarrow b W)+B R\left(t^{\prime} \rightarrow b W\right)\right] \times B R(W \rightarrow \ell \nu) \\
& \approx \sigma \times\left[B R\left(t^{\prime} \rightarrow t Z\right) \times 0.2 \times 0.99+B R\left(t^{\prime} \rightarrow b W\right)\right] \times 0.2
\end{aligned}
$$

where $\sigma$ is the single production cross-section. This indicates that, despite of $B R\left(t^{\prime} \rightarrow t Z\right)$ being larger than $B R\left(t^{\prime} \rightarrow b W\right)$ (refer to figure 8), the invisible branching ratio of the $Z$ suppresses the former contribution to the effective cross section. For light masses $m_{t^{\prime}} \lesssim$ $500 \mathrm{GeV}$, the $b j \ell^{ \pm} \mathbb{E}_{T}$ signature is therefore dominated by the $W b$ decays.

As shown in figure 8 the above decay modes are the dominant ones for heavy Higgs masses. Accordingly, to maximise the signal, we have estimated this signature using a Higgs mass of $m_{h}=1000 \mathrm{GeV}$. For a light Higgs, the branching ratios (and therefore the number of signal events) are approximately halved. One of the advantages of this process is that there is only one b-jet to be tagged and large missing transverse energy (MET) from the neutrinos could allow us to distinguish the signal from backgrounds.

In order to have an idea of the relevance of this channel at $7 \mathrm{TeV}$ LHC we have perfomed a simplified analysis. The pre-selection cuts we used are:

- Exactly one isolated lepton with transverse momentum $p_{T}>15 \mathrm{GeV}$ and rapidity $|\eta|<2.5$.

- Exactly one b-tagged jet with $p_{T}>20 \mathrm{GeV}$ and $|\eta|<3$. We assume a b-tagging efficiency of $60 \%$.

- Exactly one additional light jet with $p_{T}>20 \mathrm{GeV}$ and $|\eta|<5$.

- Minimum MET threshold: $E_{T}>30 \mathrm{GeV}$. 

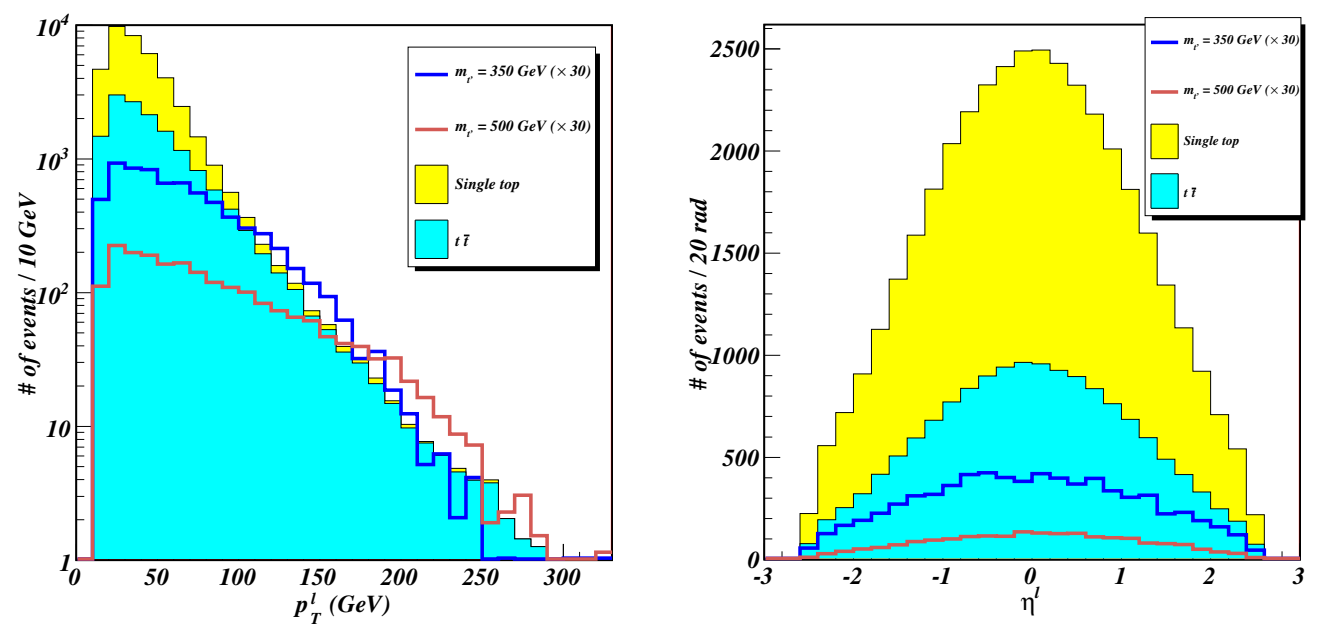

Figure 11. $b j \ell^{ \pm} \mathbb{E}_{T}$ : event distributions with respect to the $p_{T}$ (left) and rapidity (right) of the lepton at the LHC with centre of mass energy of $7 \mathrm{TeV}$ and luminosity of $10 \mathrm{fb}^{-1}$.

The distributions after pre-selection cuts are shown in figures 11, 12, 13, 14 and 15. An important feature of the distributions is that for signal events the $p_{T}$ of the light jet is relatively smaller than of the b-jet. On the other hand, the rapidity of light jet tends to be higher as compared to the b-jet. Another important feature is the distribution of the invariant mass of the bottom quark and the lepton in figure 15: this quantity has been used in the CMS search for pair production of fourth generation quarks decaying into $W b$ to remove the top background [23]. In fact, for a $b$ and lepton from a top leptonic decay, we would expect the invariant mass to be smaller than the top quark mass. In figure 15 we can see that the $t \bar{t}$ and single top backgrounds do indeed peak below $m_{\text {top }}$. For the signal, there is also a peak below the top mass coming from the $Z t$ decay modes, while the $W b$ events leak above the top mass. A cut $m_{b l}>170 \mathrm{GeV}$ could therefore help reducing the top backgrounds for small $t^{\prime}$ masses, while for large $t^{\prime}$ masses the decays are dominated by the $Z t$ mode which is severely removed by this cut. As it can be seen from figure 16, the two decay chains $t^{\prime} \rightarrow b W$ and $t^{\prime} \rightarrow t Z$ can also be distinguished from the $p_{T}$ of b-jet: the chain $t^{\prime} \rightarrow b W$ typically gives larger $p_{T}$ to the b-jet. The MET distribution can also be used for distinguishing the decay chains. The decay $t^{\prime} \rightarrow b W$ typically shows larger MET as compared to $t^{\prime} \rightarrow t Z$.

In order to further improve the significance of our results we have imposed the following secondary cuts:

- $p_{T}^{b}>100 \mathrm{GeV}$,

- $m_{b l}>170 \mathrm{GeV}$,

- $E_{T}>100 \mathrm{GeV}$.

The results of signal and backgrounds after the cuts mentioned above are shown in table 5 and in figure 15. The table shows that this mode is problematic at $7 \mathrm{TeV}$ LHC. The 

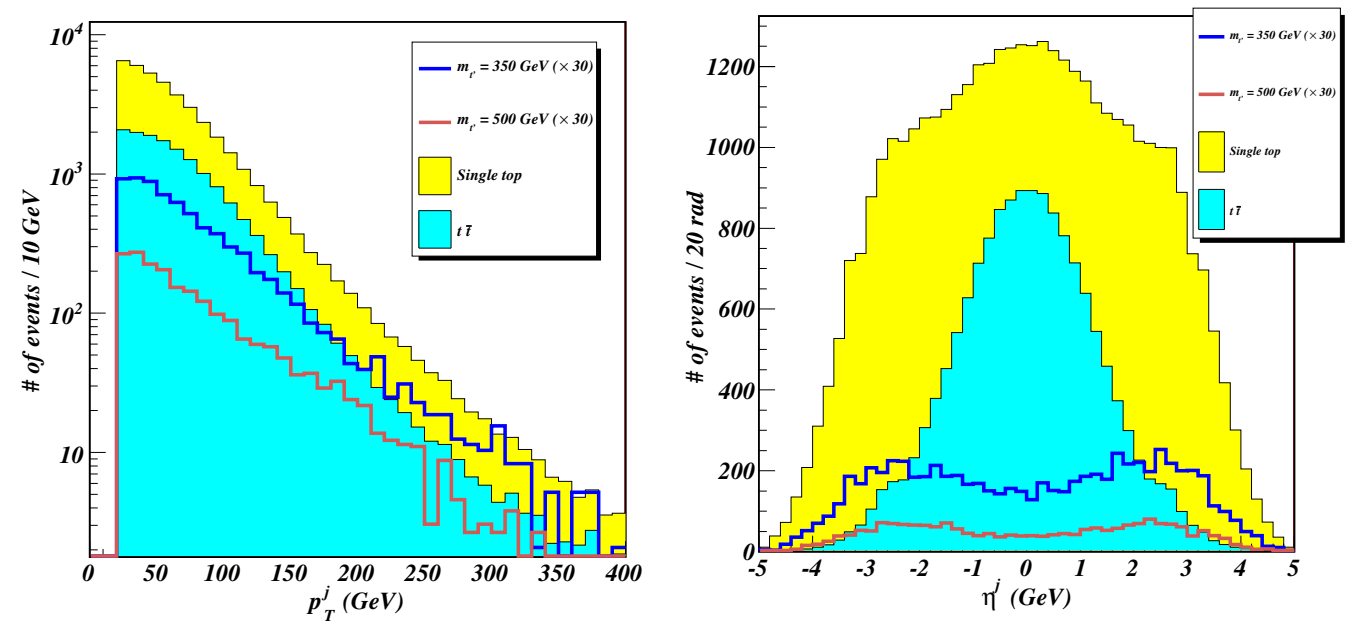

Figure 12. $b j \ell^{ \pm} \mathbb{E}_{T}$ : event distributions with respect to the $p_{T}$ (left) and rapidity (right) of the light jet at the LHC with centre of mass energy of $7 \mathrm{TeV}$ and luminosity of $10 \mathrm{fb}^{-1}$.
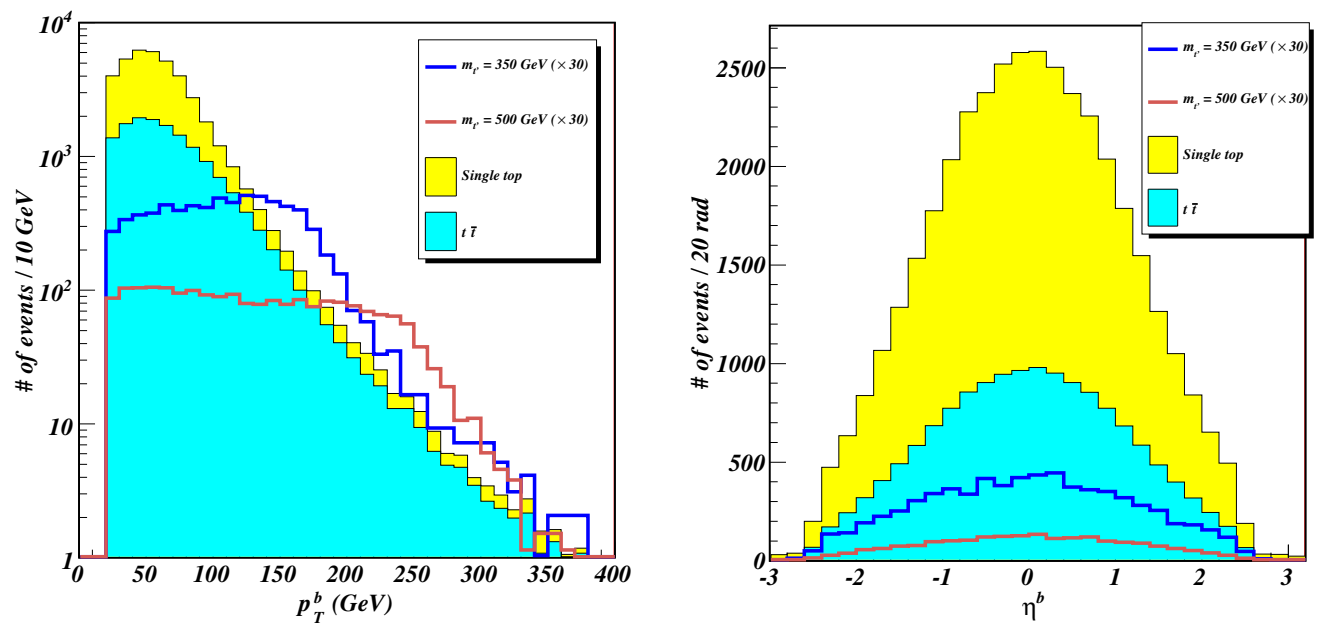

Figure 13. $b j \ell^{ \pm} \mathbb{E}_{T}$ : event distributions with respect to the $p_{T}$ (left) and rapidity (right) of the b-jet at the LHC with centre of mass energy of $7 \mathrm{TeV}$ and luminosity of $10 \mathrm{fb}^{-1}$.

reason is that the heavy vector-like quark masses explored here (up to $500 \mathrm{GeV}$ ) have a typical signature which is too close to the SM single top signal. We expect that higher mass vector-like quarks will be easier to disentangle from background because of a larger $p T$ of the b-jet and larger MET, however due to the smaller cross-section these may be accessible only at higher centre of mass energy of higher luminosities at the LHC.

\subsubsection{Signature $p p \rightarrow t^{\prime}(\rightarrow t Z) j \rightarrow b \ell^{ \pm} \ell^{\mp} \ell^{ \pm} j E_{T}$}

The trilepton channel is regarded as one one of the golden discovery channels because of the low SM background and very low probability of faking it with jets. In our case, only one tagged b-jet is required, hence it does not loose much on tagging efficiency. This signature 

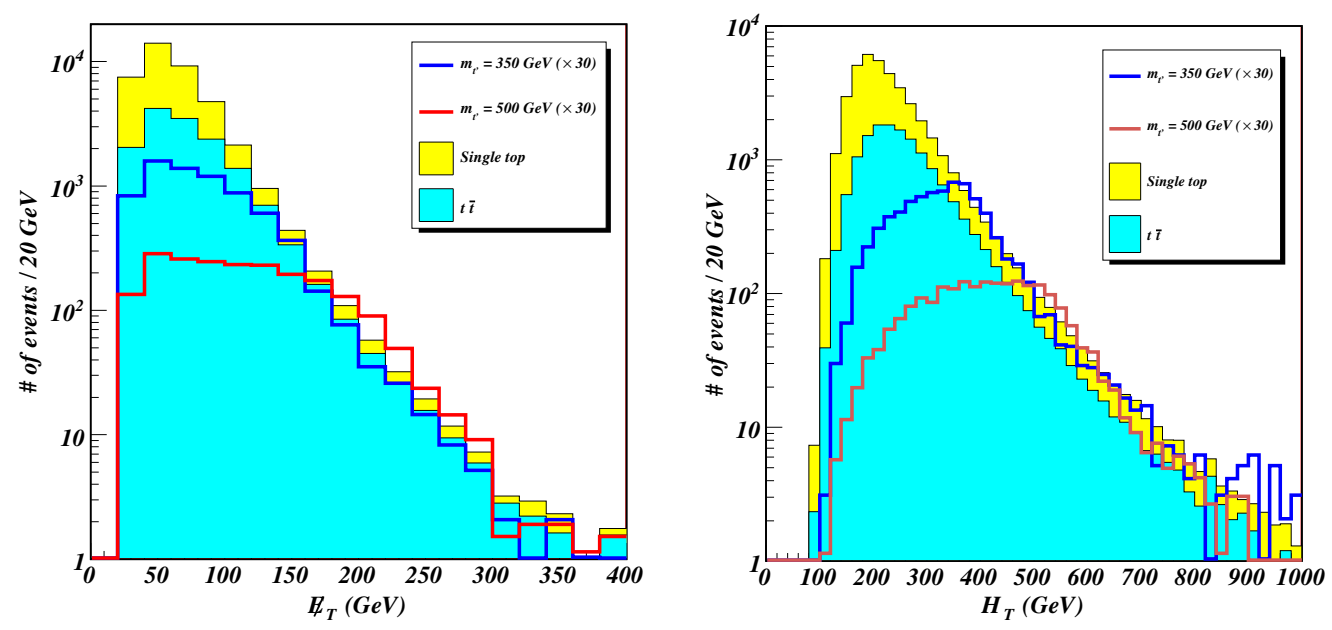

Figure 14. $b j \ell^{ \pm} E_{T}: E_{T}$ (left) and $H_{T}$ (right) distributions at the LHC with centre of mass energy of $7 \mathrm{TeV}$ and luminosity of $10 \mathrm{fb}^{-1}$.

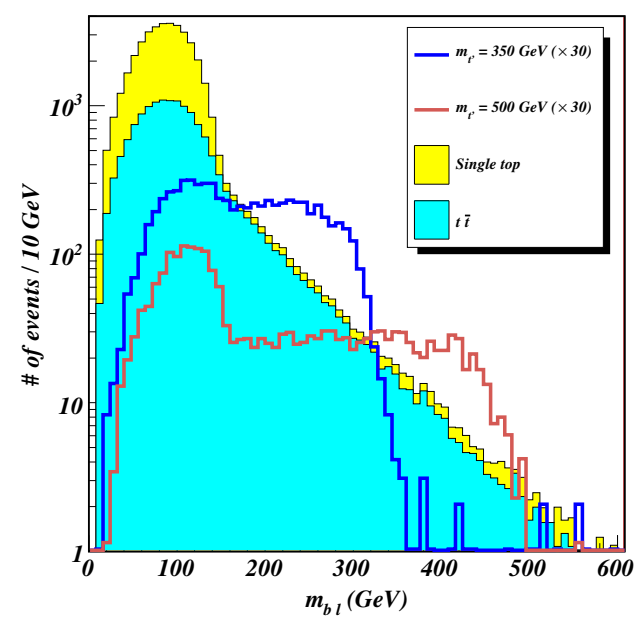

Figure 15. $b j \ell^{ \pm} \mathbb{E}_{T}$ : Invariant mass distributions of the bottom $b$ and lepton $l$ at the LHC with centre of mass energy of $7 \mathrm{TeV}$ and luminosity of $10 \mathrm{fb}^{-1}$.

is obtained from the leptonic decay of the $\mathrm{Z}$ boson: $Z \rightarrow \ell^{+} \ell^{-}$. One can also associate the whole MET as originating from the decay of $t$ and hence one can fully reconstruct the top and $t^{\prime}$. For the analysis we have used the following pre-selection cuts:

- Exactly three (two of same sign) isolated leptons with $p_{T}>20 \mathrm{GeV}$ and $|\eta|<2.5$.

- Exactly one b-tagged jet with $p_{T}>20 \mathrm{GeV}$ and $|\eta|<3$. We assume a b-tagging efficiency of $60 \%$.

- One additional light jet with $p_{T}>20 \mathrm{GeV}$ and $|\eta|<5$.

Requiring three charged lepton with sufficiently high $p_{T}$ helps in reducing the $t \bar{t}$ background where one of the lepton comes from the decay of the b-jet. 

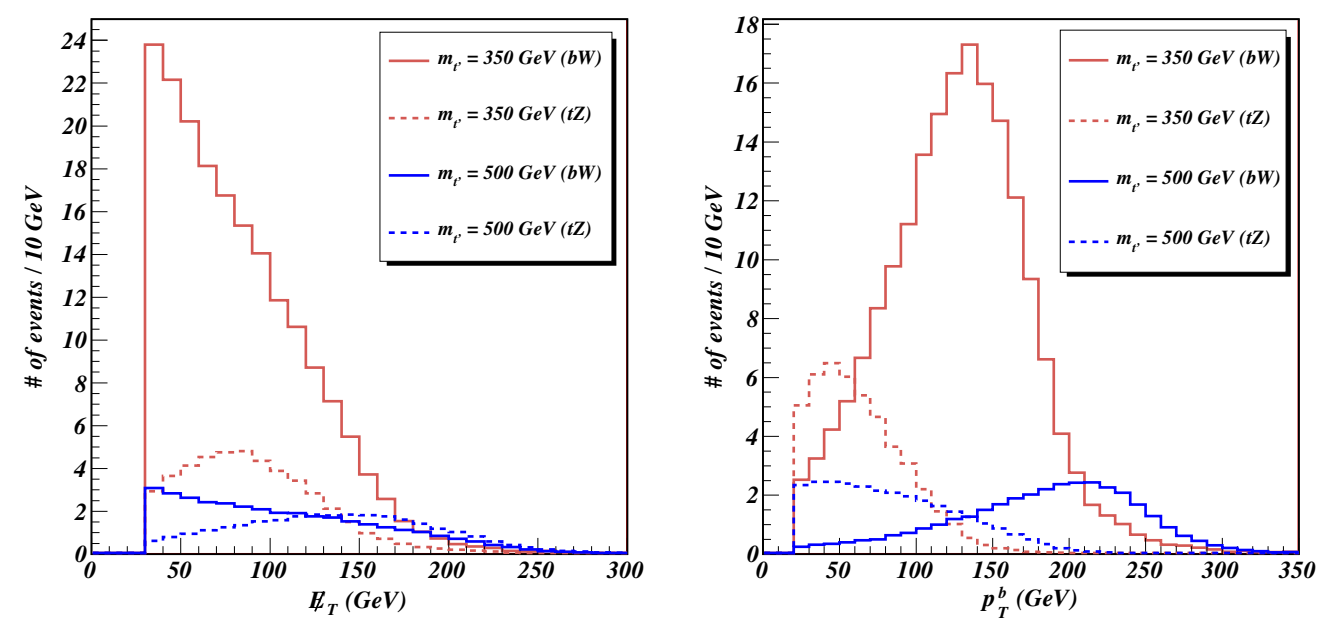

Figure 16. $b j \ell^{ \pm} E_{T}: E_{T}$ (left) and $p_{T}$ of the b-jet (right) distributions at the LHC with centre of mass energy of $7 \mathrm{TeV}$ and luminosity of $10 \mathrm{fb}^{-1}$. The contributions of the $W b$ and $Z t$ channels are plotted separately.

\begin{tabular}{|c|c|c|c|c|c|}
\hline Cut & BP 2 & BP 4 & BP 1 & BP 3 & SM \\
\hline Pre-selection & 238.9 & 69.4 & 144.8 & 41.8 & 39486 \\
$S / \sqrt{S+B}$ & 1.20 & 0.35 & 0.73 & 0.21 & - \\
\hline$p_{T}^{b}>100 \mathrm{GeV}$ & 137.9 & 52.6 & 98.6 & 25.9 & 4053.6 \\
$S / \sqrt{S+B}$ & 2.13 & 0.82 & 1.53 & 0.40 & - \\
\hline$m_{l b}>170 \mathrm{GeV}$ & 117.7 & 31.7 & - & - & 1951.1 \\
$S / \sqrt{S+B}$ & 2.59 & 0.71 & & & - \\
\hline Both previous & 90.8 & 28.0 & - & - & 992.8 \\
$S / \sqrt{S+B}$ & 2.76 & 0.86 & & & - \\
\hline$E_{T}>100 \mathrm{GeV}$ & 42.3 & 24.8 & 22.5 & 14.1 & 985.3 \\
$S / \sqrt{S+B}$ & 1.32 & 0.78 & 0.71 & 0.45 & - \\
\hline
\end{tabular}

Table 5. $b j \ell^{ \pm} E_{T}$ : Number of signal events after the cuts at the LHC with centre of mass energy of $7 \mathrm{TeV}$ and luminosity of $10 \mathrm{fb}^{-1}$.

We can reconstruct the $Z$-boson in dileptonic decay. We have three leptons in the final state and hence we can have two combinations of opposite sign dileptons. The correct combination can be chosen by selecting the one that minimise the quantity:

$$
\chi^{2}=\left(m_{Z}-m_{\ell^{+} \ell^{-}}\right)^{2} .
$$

To further reduce the backgrounds we impose that the invariant mass of the opposite sign dileptons should be close to $m_{Z}$. We have shown the opposite sign dilepton distribution in figure 17. The results of signal and background events are given in table 6. Our results show that it is indeed possible to probe $t^{\prime}$ in this channel at $7 \mathrm{TeV}$ LHC with a luminosity of $10 \mathrm{fb}^{-1}$. 


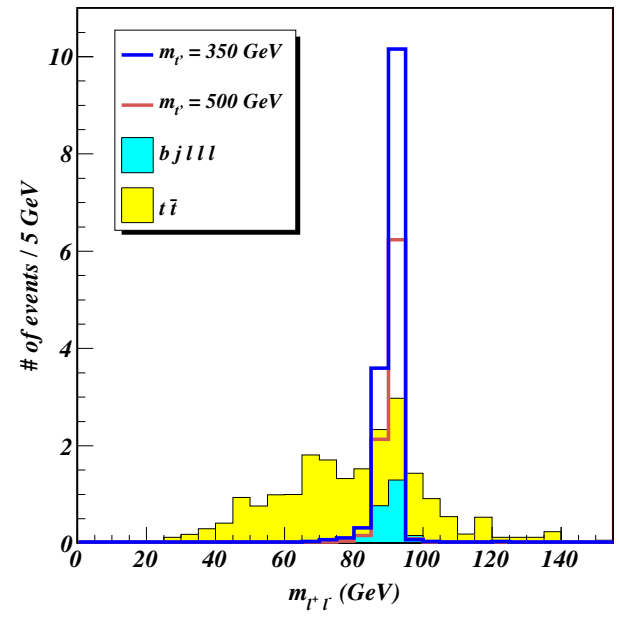

Figure 17. $b j \ell^{ \pm} \ell^{\mp} \ell^{ \pm} E_{T}$ : Opposite sign dilepton invariant mass $\left(m_{\ell^{+} \ell^{-}}\right)$distribution at the LHC with centre of mass energy of $7 \mathrm{TeV}$ and luminosity of $10 \mathrm{fb}^{-1}$. The correct combination of leptons has been selected by minimising $\chi^{2}$ as defined in the text.

\begin{tabular}{|c|c|c|c|c|c|}
\hline Cut & BP 2 & BP 4 & BP 1 & BP 3 & SM \\
\hline Pre-selection & 14.4 & 8.8 & 6.5 & 4.2 & 20.7 \\
\hline$\left|m_{\ell^{+} \ell^{-}}-m_{Z}\right|<5 \mathrm{GeV}$ & 13.6 & 8.3 & 5.7 & 4 & 3.8 \\
$S / \sqrt{S+B}$ & 3.3 & 2.4 & 1.8 & 0.84 & - \\
\hline
\end{tabular}

Table 6. $b j \ell^{ \pm} \ell^{\mp} \ell^{ \pm} \mathbb{E}_{T}$ : Number of signal events after the cuts at the LHC with centre of mass energy of $7 \mathrm{TeV}$ and luminosity of $10 \mathrm{fb}^{-1}$.

\subsubsection{Signature $p p \rightarrow t^{\prime}(\rightarrow t H) j \rightarrow b \bar{b} b \quad j \ell^{ \pm} E_{T}$}

This signature requires the presence of three tagged b-jets. This makes the detection of this signature challenging, especially at low energy and luminosities. The largest backgrounds to this channel might come from $t \bar{t}$ production where one of the light jets is misidentified with a b-jet.

The additional problem in this channel comes because of the presence of three b-jets and hence, due to combinatorial background problems, it might be difficult to reconstruct the Higgs boson.

\subsubsection{Signature $p p \rightarrow t^{\prime}(\rightarrow t Z) j \rightarrow b j j j \ell^{ \pm} E_{T}$}

Although this channel will probably have the highest effective cross-section (when we take BRs into account), one has to face the problem of the $Z$ reconstruction. It would be possible to reconstruct the $Z$ invariant mass using $Z \rightarrow j j$, however the reconstruction might not be very good because of the presence of an additional light jet in the event which will give rise to combinatorial backgrounds. Even if we were able to reconstruct Z, we may not be able to reduce backgrounds with $W \rightarrow j j$ as this too occurrs around the $Z$ mass and the energy resolution with jets is typically not very good. Such backgrounds could possibly come from $t \bar{t}$ and it would be quite challenging to reduce them. 

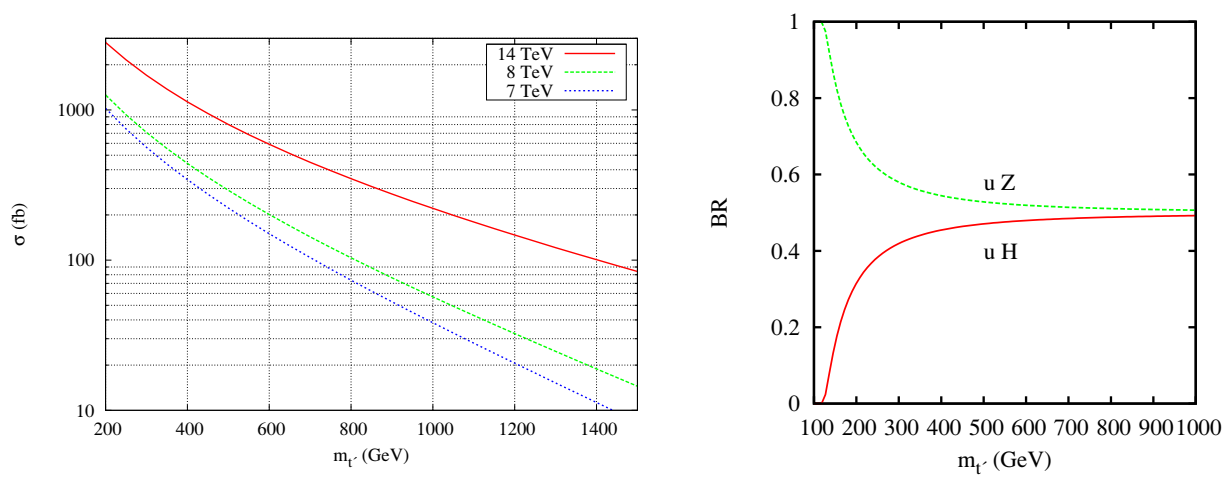

Figure 18. Single production cross-section (left) and branching ratios (right) of $t^{\prime}$ as a function of $m_{t^{\prime}}$ for $\sin \theta^{R}=0$. The remaining parameters are fixed at the benchmark values in table 3 .

\subsubsection{Signature $p p \rightarrow t^{\prime}(\rightarrow j H) j \rightarrow j j \bar{b} b$, in the case $\sin \theta^{R}=0$.}

The single production of $t^{\prime}$ in association with light jet has also been considered in [43] where they considered $t^{\prime}$ decays to be:

$$
t^{\prime} \rightarrow W / Z+j
$$

with $\mathrm{j}=\mathrm{u}, \mathrm{d}$ and argued that this is the most general scenario. Here we showed that these modes are quite suppressed compared to decays into the third generation, due to the tight bounds from precision flavour measurements that limit the size of the couplings to light quarks.

The situation is different only for $\sin \theta^{R}=0$ : in this case, the heavy top $t^{\prime}$ decouples from the top, therefore it can only decay into light quarks. An important difference is the presence of the $h j$ channel, that has not been considered in [43]. In our specific case, namely a non-standard doublet, the decays mediated by the $W$ to light quarks are suppressed by the mass of the quark, therefore they are negligible. Furthermore, as we can see from figure 18 , the mode $h j$ dominates with respect to $Z j$. The production cross sections are very similar to the previous case under consideration, as it can be seen from figure 18 .

In the rest of this section, we will focus on the decay mode

$$
t^{\prime} \rightarrow h+j .
$$

Considering the dominant decay of $h \rightarrow b \bar{b}$, we will get a signature of $p p \rightarrow t^{\prime} j \rightarrow j j b \bar{b}$. To study the $b \bar{b} j j$ final state, we impose the following pre-selection cuts:

- Two light jets with $p_{T}>20 \mathrm{GeV}$ and $|\eta|<5$.

- Two b-tagged jets with $p_{T}>20 \mathrm{GeV}$ and $|\eta|<3$.

For this signature, we considered the background coming from $Z$ and $h+$ jets, with the bosons decaying into a $b \bar{b}$ pair, that has been generated with ALPGEN. In figure 19 we plotted the $p_{T}$ distribution of the highest $p_{T}$ jet for signal and backgrounds. As can be seen from this distribution one can reduce the backgrounds by using a hard cut on the $p_{T}$ 


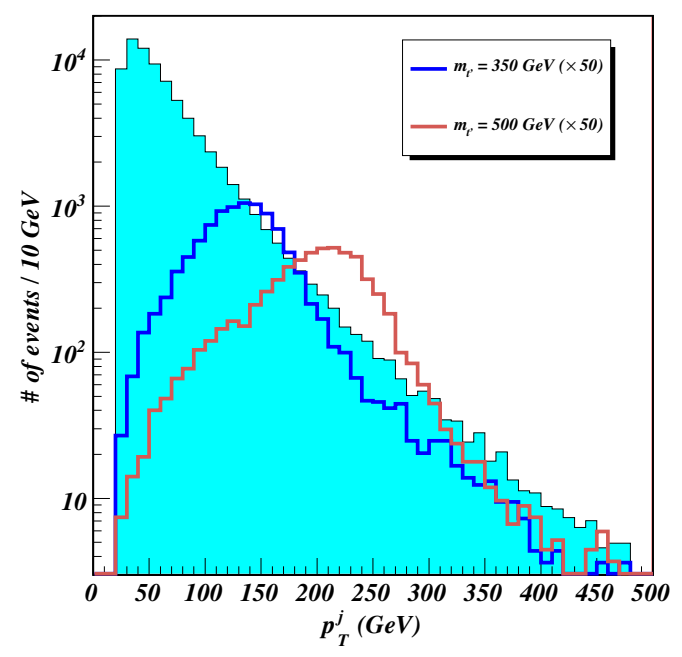

Figure 19. $b \bar{b} j j: p_{T}$ of the higest $p_{T}$ light jet at the LHC with centre of mass energy of $7 \mathrm{TeV}$ and lumionsity of $10 \mathrm{fb}^{-1}$.
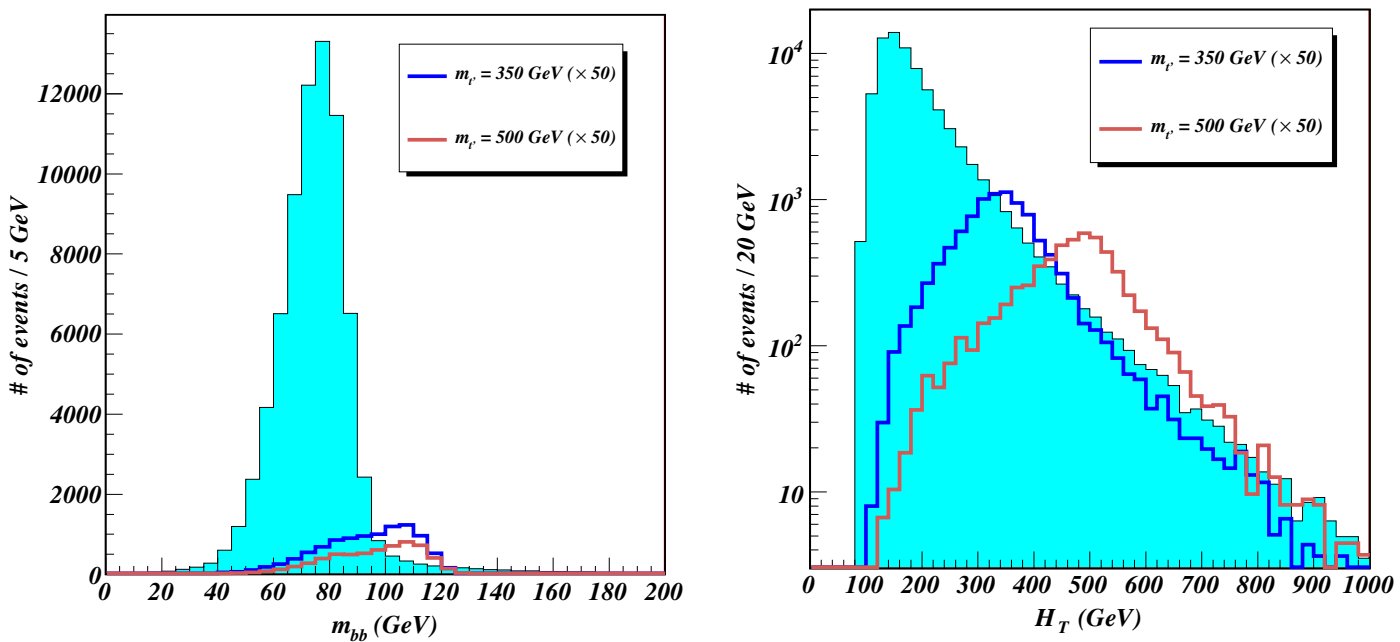

Figure 20. $b \bar{b} j j: m_{b \bar{b}}$ and $H_{T}$ distributions at the LHC with centre of mass energy of $7 \mathrm{TeV}$ and lumionsity of $10 \mathrm{fb}^{-1}$.

of the jet. In figure 20 we also plotted the invariant mass $m_{b \bar{b}}$ and total transverse energy $H_{T}$ distributions, where $H_{T}$ is defined as the scalar sum of the transverse $p_{T}$ of the visible particles (jets and b-jets in our case). We can therefore improve the significance of this signature by imposing additional cuts:

- Cut A: $p_{T}^{j}>100 \mathrm{GeV}$. Hard cut on the $p_{T}$ of the highest $p_{T}$ jet.

- Cut B: $\left|m_{b \bar{b}}-m_{h}\right|<25 \mathrm{GeV}$.

- Cut C: Total transverse energy $H_{T}>300 \mathrm{GeV}$.

- Cut D: Total transverse energy $H_{T}>350 \mathrm{GeV}$. 


\begin{tabular}{|c|c|c|c|}
\hline Cut & BP 1-A & BP 2-A & SM \\
\hline Pre-selection & 350.1 & 206.4 & 74500 \\
$S / \sqrt{S+B}$ & 1.3 & 0.75 & - \\
\hline Cut A & 278.4 & 193.8 & 11440 \\
$S / \sqrt{S+B}$ & 2.6 & 1.8 & - \\
\hline Cut B & 166.9 & 128.2 & 498.4 \\
$S / \sqrt{S+B}$ & 6.5 & 5.1 & - \\
\hline Cut C & 151.6 & 126.1 & 341.7 \\
$S / \sqrt{S+B}$ & 6.8 & 5.8 & - \\
\hline Cut D & 106.4 & 120.3 & 219.1 \\
$S / \sqrt{S+B}$ & 5.9 & 6.5 & - \\
\hline
\end{tabular}

Table 7. $b \bar{b} j j$ : No of signal events after the cuts for LHC with CM energy of $7 \mathrm{TeV}$ and Luminosity of $10 \mathrm{fb}^{-1}$.

In table 7 we have shown the effects of the above cuts on signal and backgrounds. These results show that the backgrounds can be reduced and a a good significance achieved on the signal.

\section{Conclusions}

We have discussed in detail new vector-like quarks which can mix with the standard model quarks without conflicting with current experimental limits. In particular we focused on a non standard doublet that contains a top partner and an exotic quark with electric charge $5 / 3$. This case is motivated from models of composite Higgs, where such fermions are the lightest top partners, and from the less severe bounds from precision observables on the general parameter space. In this paper, we discussed in detail the full flavour structure of the model including mixing with the light generations, and we studied the bounds on such mixing from flavour observables in the D meson sector and atomic parity violation measurements. We also studied their suppressed loop contribution to the mixing in the Kaon and B meson sectors, where large effects may appear especially in the CP violating observables. While all other effects are smaller than the theoretical uncertainty on the form factors, we found a potentially large effect on the phase of the $B_{s}$ mixing amounting to a correction up to $\pm 150 \%$ of the Standard Model phase. We also considered the new bounds from the recent data at the LHC with a luminosity of up to $1 \mathrm{fb}^{-1}$ : the bounds are lower than the nominal ones due to the reduced branching ratio in the final states analysed by the collaborations, therefore masses as low as 300 or $380 \mathrm{GeV}$ are still allowed.

In the second part of the paper, we considered some LHC signatures of this scenario. In particular, we focused on single production of the heavy top partner due to the flavour violation couplings of the $Z$ boson: this channel is dominated by up-quark initiated processes. Notwithstanding the suppressed coupling, the cross section is enhanced by the large PDF's of the valence ups, and it can dominate over the pair production channel at large masses, about $\sim 400 \mathrm{GeV}$. We performed a detailed simulation of signals and backgrounds 
for a few benchmark points. For generic parameters, the preferred decay final states involve $W, Z$ and Higgs plus third generation quarks. The most promising channels contain at least one lepton in the final state from the top or $W$ decays. The main background for these channels is given by events containing top quarks. We identified the most promising final state as $b j \ell^{ \pm} \ell^{\mp} \ell^{ \pm} \mathbb{E}_{T}$. For light $t^{\prime}$ masses, however, the top backgrounds have very similar kinematic structure as the $t^{\prime}$ signal, therefore it is very hard to disentangle the signal events and an integrated luminosity of $10 \mathrm{fb}^{-1}$ at $7 \mathrm{TeV}$ of centre of mass energy may not be enough for the discovery. The LHC with a higher centre of mass energy and good luminosity can improve the situation, especially by accessing higher mass cases where the distinction is possible if enough events are collected.

Finally, in the case of no mixing with the top quark, the $t^{\prime}$ decays mostly into a light Higgs plus a jet: this channel would generate the final state $b \bar{b} j j$. By imposing cuts on the invariant mass of the b-tagged jet pair and on the total transverse energy, it is possible to reduce the backgrounds and discover this channel with luminosities below $10 \mathrm{fb}^{-1}$.

Our exploration showed that the phenomenology of vector-like fermions can be very rich, and many other channels, not considered here, deserve further detailed investigation. Moreover, a generic model independent analysis of the parameter space, not biased by any specific model, is crucial at this point of the LHC as it would allow us to fully exclude this possibility or, in case of excesses in the data, it would offer hints on the kind of new physics at the $\mathrm{TeV}$ scale.

\section{Acknowledgments}

NG would like to thank IPNL, Lyon, France and National Central University (NCU), Chung-Li, Taiwan for the hospitality where part of this work was done. Y.O. and L.P. would like to thank W.S. Hou and C.-P. Yuan for useful discussions at "Focus Workshop on Heavy Quarks at LHC" held at National Taiwan University. The research of Y.O. is supported in part by the Grant-in-Aid for Science Research, Japan Society for the Promotion of Science (JSPS), No. 20244037 and No. 22244031. The work of N.G. is supported by a grants from Department of Science \& Technology (DST), India under project no. SR/S2/HEP-09/10 and University Grants Commission (UGC), India under project No. 38-58/2009(SR).

\section{A Expansion of the CKM matrix}

We give in the following a systematic expansion of the CKM matrix in our modified framework, including the extra vector-like top partner. The mixing angles in the left-handed sector are small, and we can safely assume that the main structure of the CKM matrix is coming from the SM Yukawas, therefore $\tilde{V}_{C K M}$ is very close to the measured CKM matrix. A simple way to estimate the impact of the mixing in the left-handed sector is to use the hierarchy in $\tilde{V}_{C K M}$ and the quark masses in terms of the Cabibbo angle $\lambda=\sin \theta_{12}=0.2252$, where $\theta_{12}$ is the mixing angle between the first and second generation. It is well known that 
the SM CKM matrix is hierarchical: we will use here the Wolfenstein parameterisation [13]

$$
\tilde{V}_{C K M}=\left(\begin{array}{ccc}
1-\lambda^{2} / 2 & \lambda & A \lambda^{3}(\rho-i \eta) \\
-\lambda & 1-\lambda^{2} / 2 & A \lambda^{2} \\
A \lambda^{3}(1-\rho-i \eta) & -A \lambda^{2} & 1
\end{array}\right)+\mathcal{O}\left(\lambda^{4}\right) \sim\left(\begin{array}{ccc}
\mathcal{O}(1) & \mathcal{O}(\lambda) & \mathcal{O}\left(\lambda^{3}\right) \\
\mathcal{O}(\lambda) & \mathcal{O}(1) & \mathcal{O}\left(\lambda^{2}\right) \\
\mathcal{O}\left(\lambda^{3}\right) & \mathcal{O}\left(\lambda^{2}\right) & \mathcal{O}(1)
\end{array}\right)
$$

The hierarchy in the quark masses can also be related to the Cabibbo angle, $\frac{m_{c}}{m_{t}} \sim \mathcal{O}\left(\lambda^{3}\right)$ and $\frac{m_{u}}{m_{t}} \sim \mathcal{O}\left(\lambda^{7}\right)$, therefore one can also determine a hierarchy in the matrix $V_{L}$ :

$$
V_{L} \sim\left(\begin{array}{ccc}
\mathcal{O}(1) & \mathcal{O}\left(\lambda^{4}\right) & \mathcal{O}\left(\lambda^{7}\right) \\
\mathcal{O}\left(\lambda^{4}\right) & \mathcal{O}(1) & \mathcal{O}\left(\lambda^{3}\right) \\
\mathcal{O}\left(\lambda^{7}\right) & \mathcal{O}\left(\lambda^{3}\right) & \mathcal{O}(1)
\end{array}\right)
$$

Note that the powers of $\lambda$ only take into account the suppression coming from the quark masses, and that additional suppressions will come from the small mixing with the vector fermions, as discussed in section 3.7. Using the expansion in powers of $\lambda$, we can order the contributions to the elements of $V_{C K M}=V_{L}^{\dagger} \cdot V_{C K M}^{\prime}$. Moreover, we will explicitly indicate the phases of the new Yukawas $x_{1}$ and $x_{2}$ to make clear where the new phases enter while the SM phase is hidden in the standard CKM elements $V_{q q^{\prime}}$ :

$$
\begin{aligned}
V_{C K M}^{u d} & =V_{u d}\left[\lambda^{0}\right]+e^{i\left(\beta_{2}-\beta_{1}\right)}\left|V_{L}^{21}\right| V_{c d}\left[\lambda^{5}\right]+e^{-i \beta_{1}}\left|V_{L}^{31}\right| V_{t d}\left[\lambda^{10}\right], \\
V_{C K M}^{u s} & =V_{u s}\left[\lambda^{2}\right]+e^{i\left(\beta_{2}-\beta_{1}\right)}\left|V_{L}^{21}\right| V_{c s}\left[\lambda^{4}\right]+e^{-i \beta_{1}}\left|V_{L}^{31}\right| V_{t s}\left[\lambda^{9}\right], \\
V_{C K M}^{u b} & =V_{u b}\left[\lambda^{3}\right]+e^{i\left(\beta_{2}-\beta_{1}\right)}\left|V_{L}^{21}\right| V_{c b}\left[\lambda^{6}\right]+e^{-i \beta_{1}}\left|V_{L}^{31}\right| V_{t b}\left[\lambda^{7}\right], \\
V_{C K M}^{c d} & =V_{c d}\left[\lambda^{2}\right]-e^{-i\left(\beta_{2}-\beta_{1}\right)}\left|V_{L}^{12}\right| V_{u d}\left[\lambda^{4}\right]+e^{-i \beta_{2}}\left|V_{L}^{32}\right| V_{t d}\left[\lambda^{6}\right], \\
V_{C K M}^{c s} & =V_{c s}\left[\lambda^{0}\right]+\left(-e^{-i\left(\beta_{2}-\beta_{1}\right)}\left|V_{L}^{12}\right| V_{u s}+e^{-i \beta_{2}}\left|V_{L}^{32}\right| V_{t s}\right)\left[\lambda^{4}\right], \\
V_{C K M}^{c b} & =V_{c b}\left[\lambda^{2}\right]+e^{-i \beta_{2}}\left|V_{L}^{32}\right| V_{t b}\left[\lambda^{3}\right]-e^{-i\left(\beta_{2}-\beta_{1}\right)}\left|V_{L}^{12}\right| V_{u b}\left[\lambda^{7}\right], \\
V_{C K M}^{t d} & =V_{L}^{33} V_{t d}\left[\lambda^{3}\right]-e^{i \beta_{2}}\left|V_{L}^{23}\right| V_{c d}\left[\lambda^{4}\right]-e^{i \beta_{1}}\left|V_{L}^{13}\right| V_{u d}\left[\lambda^{7}\right], \\
V_{C K M}^{t s} & =V_{L}^{33} V_{t s}\left[\lambda^{2}\right]-e^{i \beta_{2}}\left|V_{L}^{23}\right| V_{c s}\left[\lambda^{3}\right]-e^{i \beta_{1}}\left|V_{L}^{13}\right| V_{u s}\left[\lambda^{8}\right], \\
V_{C K M}^{t b} & =V_{L}^{33} V_{t b}\left[\lambda^{0}\right]-e^{i \beta_{2}}\left|V_{L}^{23}\right| V_{c b}\left[\lambda^{5}\right]-e^{i \beta_{1}}\left|V_{L}^{13}\right| V_{t d}\left[\lambda^{10}\right] ;
\end{aligned}
$$

in square brackets we give the power counting in $\lambda$ for each term. Analogously, one can calculate the couplings of $t^{\prime}$ to the $W$ (taking into account that $V_{L}^{14} \sim \mathcal{O}\left(\lambda^{7}\right), V_{L}^{24} \sim \mathcal{O}\left(\lambda^{3}\right)$ and $\left.V_{L}^{34} \sim \mathcal{O}(1)\right)$ :

$$
\begin{aligned}
V_{C K M}^{t^{\prime} d} & =V_{L}^{34} V_{t d}\left[\lambda^{3}\right]+e^{i \beta_{2}}\left|V_{L}^{24}\right| V_{c d}\left[\lambda^{4}\right]+e^{i \beta_{1}}\left|V_{L}^{14}\right| V_{u d}\left[\lambda^{7}\right], \\
V_{C K M}^{t^{\prime} s} & =V_{L}^{34} V_{t s}\left[\lambda^{2}\right]+e^{i \beta_{2}}\left|V_{L}^{24}\right| V_{c s}\left[\lambda^{3}\right]+e^{i \beta_{1}}\left|V_{L}^{14}\right| V_{u s}\left[\lambda^{8}\right], \\
V_{C K M}^{t^{\prime} b} & =V_{L}^{34} V_{t b}\left[\lambda^{0}\right]+e^{i \beta_{2}}\left|V_{L}^{24}\right| V_{c b}\left[\lambda^{5}\right]+e^{i \beta_{1}}\left|V_{L}^{14}\right| V_{t d}\left[\lambda^{10}\right] .
\end{aligned}
$$

\section{B Notation for the meson mixing}

The $K^{0}-\bar{K}^{0}$ mixing can be calculated in our model with an extra non-standard doublet by a simple generalisation of the usual formulas, by including the effect of the $t^{\prime}$ quark 
as shown in figure 2. The effective Hamiltonian describing $K^{0}-\bar{K}^{0}$ mixing is a simple extension of the standard one and it is given by

$$
\mathcal{H}_{\mathrm{eff}}=\frac{G_{F}}{\sqrt{2}} \frac{\alpha}{4 \pi \sin ^{2} \theta_{W}} \sum_{i, j=c, t, t^{\prime}} \eta_{i j} \xi^{i} \xi^{j} E\left(x_{i}, x_{j}\right)\left[\bar{s} \gamma_{\mu} P_{L} d\right]\left[\bar{s} \gamma^{\mu} P_{L} d\right]
$$

with

$$
\begin{aligned}
E\left(x_{i}, x_{j}\right)= & x_{i} x_{j}\left[-\frac{3}{4\left(1-x_{i}\right)\left(1-x_{j}\right)}+\frac{\log x_{i}}{\left(x_{i}-x_{j}\right)\left(1-x_{i}\right)^{2}}\left(1-2 x_{i}+\frac{x_{i}^{2}}{4}\right)\right. \\
& \left.+\frac{\log x_{j}}{\left(x_{j}-x_{i}\right)\left(1-x_{j}\right)^{2}}\left(1-2 x_{j}+\frac{x_{j}^{2}}{4}\right)\right]
\end{aligned}
$$

for $i \neq j$. For $i=j$ the Inami-Lim function $E\left(x_{i}, x_{j}\right)$ becomes

$$
E\left(x_{i}, x_{i}\right)=\frac{x_{i}}{\left(1-x_{i}\right)^{2}}\left[1-\frac{11 x_{i}}{4}+\frac{x_{i}^{2}}{4}-\frac{3 x_{i}^{2} \log x_{i}}{2\left(1-x_{i}\right)}\right]
$$

where $\eta_{i j}$ are QCD corrections to the Inami-Lim functions, $\xi^{i}=V_{C K M}^{i s} V_{C K M}^{*, i d}$ and $x_{i}=$ $m_{i}^{2} / m_{W}^{2} ; V_{C K M}^{\dagger} V_{C K M}$ leads to the quadrangle condition $\xi^{u}+\xi^{c}+\xi^{t}+\xi^{t^{\prime}}=0$. We checked these formulas by calculating the loop box diagrams in Feynman-'t Hooft gauge: in the mass eigenstate basis, the couplings of the $W$ bosons are given in eq. (2.23).

The hadronic matrix element entering the $\bar{K}^{0}-K^{0}$ mixing is

$$
\left\langle K^{0}\left|\left[\bar{s} \gamma_{\mu} P_{L} d\right]\left[\bar{s} \gamma^{\mu} P_{L} d\right]\right| \bar{K}^{0}\right\rangle=\frac{2}{3} f_{K}^{2} B_{K} m_{K}^{2}
$$

where $f_{K} \simeq 160 \mathrm{MeV}$ is the Kaon decay constant, its mass is $m_{K}=497.614 \pm 0.024 \mathrm{MeV}$ and $B_{K}=0.725 \pm 0.026$ is the bag parameter. In the non-SM doublet model, the mixing matrix element $M_{12}$ becomes

$$
M_{12}=\frac{1}{2 m_{K}}\left\langle K^{0}\left|\mathcal{H}_{\mathrm{eff}}\right| \bar{K}^{0}\right\rangle=\frac{1}{3} f_{K}^{2} B_{K} m_{K} \frac{G_{F}}{\sqrt{2}} \frac{\alpha}{4 \pi \sin ^{2} \theta_{W}} \sum_{i, j=c, t, t^{\prime}} \eta_{i j} \xi^{i} \xi^{j} E\left(x_{i}, x_{j}\right)
$$

Here we are interested in two CP violating quantities: the mass difference $\Delta m_{K}$ between the two mass eigenstates $K_{L / S}$, and the direct $\mathrm{CP}$ violation parameter $\epsilon_{K}$, defined in terms of the matrix element as

$$
\begin{aligned}
\Delta m_{K} & \equiv m_{K_{L}}-m_{K_{S}}=2\left|M_{12}\right| \simeq 2 \operatorname{Re} M_{12}, \\
\epsilon_{K} & \simeq \frac{e^{i \pi / 4}}{\sqrt{2} \Delta m_{K}} I m M_{12} .
\end{aligned}
$$

With the non-SM doublet we consider, $\Delta m_{K}$ is given by

$$
\Delta m_{K}=\frac{G_{F}}{\sqrt{2}} \frac{\alpha f_{K}^{2} m_{K}}{6 \pi \sin ^{2} \theta_{W}} \sum_{i, j=c, t, t^{\prime}}\left|\eta_{i j} \xi^{i} \xi^{j} E\left(x_{i}, x_{j}\right)\right|
$$


the $\xi^{i}$ can be expressed as (where we show the ordering in powers of $\lambda$ )

$$
\begin{aligned}
\xi^{c} & \sim V_{c s} V_{c d}^{*}\left[\lambda^{2}\right]-e^{i\left(\beta_{2}-\beta_{1}\right)}\left|V_{L}^{12}\right| V_{c s} V_{u d}^{*}\left[\lambda^{4}\right], \\
\xi^{t} & \sim\left|V_{L}^{33}\right|^{2} V_{t s} V_{t d}^{*}\left[\lambda^{5}\right]-V_{L}^{33}\left|V_{L}^{23}\right|\left(e^{-i \beta_{2}} V_{t s} V_{c d}^{*}+e^{i \beta_{2}} V_{c s} V_{t d}^{*}\right)\left[\lambda^{6}\right]+\left|V_{L}^{23}\right|^{2} V_{c s} V_{c d}^{*}\left[\lambda^{7}\right], \\
\xi^{t^{\prime}} & \sim\left|V_{L}^{34}\right|^{2} V_{t s} V_{t d}^{*}\left[\lambda^{5}\right]+e^{i \beta_{2}} V_{L}^{34}\left|V_{L}^{24}\right| V_{c s} V_{t d}^{*}\left[\lambda^{6}\right]+e^{-i \beta_{2}} V_{L}^{34}\left|V_{L}^{24}\right| V_{t s} V_{c d}^{*}\left[\lambda^{6}\right] .
\end{aligned}
$$

The formulas in this section can be easily generalised to the case of $B_{s}$ and $B_{d}$ mixing: it is enough to replace the $s$ quark with a $b$ and the $d$ quark with a $s$ or $d$ respectively. The relevant CKM entries in the two cases will be

$$
\xi_{s}^{i}=V_{C K M}^{i b} V_{C K M}^{*, i s} \quad \text { and } \quad \xi_{d}^{i}=V_{C K M}^{i b} V_{C K M}^{*, i d}
$$

with $i=u, c, t, t^{\prime}$.

Open Access. This article is distributed under the terms of the Creative Commons Attribution License which permits any use, distribution and reproduction in any medium, provided the original author(s) and source are credited.

\section{References}

[1] B. Grinstein, M. Redi and G. Villadoro, Low Scale Flavor Gauge Symmetries, JHEP 11 (2010) 067 [arXiv: 1009.2049] [INSPIRE].

[2] D. Guadagnoli, R.N. Mohapatra and I. Sung, Gauged Flavor Group with Left-Right Symmetry, JHEP 04 (2011) 093 [arXiv:1103.4170] [INSPIRE].

[3] N. Arkani-Hamed, A. Cohen, E. Katz and A. Nelson, The Littlest Higgs, JHEP 07 (2002) 034 [hep-ph/0206021] [INSPIRE].

[4] T. Han, H.E. Logan, B. McElrath and L.-T. Wang, Phenomenology of the little Higgs model, Phys. Rev. D 67 (2003) 095004 [hep-ph/0301040] [INSPIRE].

[5] M. Perelstein, M.E. Peskin and A. Pierce, Top quarks and electroweak symmetry breaking in little Higgs models, Phys. Rev. D 69 (2004) 075002 [hep-ph/0310039] [INSPIRE].

[6] M. Schmaltz and D. Tucker-Smith, Little Higgs review, Ann. Rev. Nucl. Part. Sci. 55 (2005) 229 [hep-ph/0502182] [INSPIRE].

[7] R. Contino, L. Da Rold and A. Pomarol, Light custodians in natural composite Higgs models, Phys. Rev. D 75 (2007) 055014 [hep-ph/0612048] [INSPIRE].

[8] B.A. Dobrescu and C.T. Hill, Electroweak symmetry breaking via top condensation seesaw, Phys. Rev. Lett. 81 (1998) 2634 [hep-ph/9712319] [INSPIRE].

[9] R. Chivukula, B.A. Dobrescu, H. Georgi and C.T. Hill, Top quark seesaw theory of electroweak symmetry breaking, Phys. Rev. D 59 (1999) 075003 [hep-ph/9809470] [InSPIRE].

[10] H.-J. He, C.T. Hill and T.M. Tait, Top quark seesaw, vacuum structure and electroweak precision constraints, Phys. Rev. D 65 (2002) 055006 [hep-ph/0108041] [INSPIRE].

[11] C.T. Hill and E.H. Simmons, Strong dynamics and electroweak symmetry breaking, Phys. Rept. 381 (2003) 235 [Erratum ibid. 390 (2004) 553] [hep-ph/0203079] [INSPIRE]. 
[12] C. Anastasiou, E. Furlan and J. Santiago, Realistic Composite Higgs Models, Phys. Rev. D 79 (2009) 075003 [arXiv:0901.2117] [InSPIRE].

[13] Particle Data Group collaboration, K. Nakamura et al., Review of particle physics, J. Phys. G 37 (2010) 075021 [inSPIRE].

[14] G. Cacciapaglia, A. Deandrea, D. Harada and Y. Okada, Bounds and Decays of New Heavy Vector-like Top Partners, JHEP 11 (2010) 159 [arXiv:1007.2933] [INSPIRE].

[15] F. del Aguila, M. Pérez-Victoria and J. Santiago, Effective description of quark mixing, Phys. Lett. B 492 (2000) 98 [hep-ph/0007160] [INSPIRE].

[16] F. del Aguila, M. Pérez-Victoria and J. Santiago, Observable contributions of new exotic quarks to quark mixing, JHEP 09 (2000) 011 [hep-ph/0007316] [INSPIRE].

[17] J. Alwall et al., Is $V_{t b} \simeq 1$ ?, Eur. Phys. J. C 49 (2007) 791 [hep-ph/0607115] [INSPIRE].

[18] F. del Aguila et al., Collider aspects of flavour physics at high $Q$, Eur. Phys. J. C 57 (2008) 183 [arXiv:0801.1800] [InSPIRE].

[19] P. Bamert, C.P. Burgess, J.M. Cline, D. London and E. Nardi, $R(b)$ and new physics: A Comprehensive analysis, Phys. Rev. D 54 (1996) 4275 [hep-ph/9602438] [INSPIRE].

[20] K. Agashe, R. Contino, L. Da Rold and A. Pomarol, A Custodial symmetry for Z $b \bar{b}$, Phys. Lett. B 641 (2006) 62 [hep-ph/0605341] [INSPIRE].

[21] G. Panico and A. Wulzer, The Discrete Composite Higgs Model, JHEP 09 (2011) 135 [arXiv: 1106.2719] [INSPIRE].

[22] CMS collaboration, Search for a Top-like Quark Decaying To A Top Quark And a Z boson in pp Collisions at $\sqrt{s}=7 \mathrm{TeV}$, CMS preprint PAS EXO-11-005, July 13 (2011), http://cdsweb.cern.ch/record/1367130/files/EXO-11-005-pas.pdf.

[23] CMS collaboration, Search for pair production of a fourth-generation $t^{\prime}$ quark in the lepton-plus-jets channel with the CMS experiment, CMS preprint PAS EXO-11-051, July 22 (2011), http://cdsweb.cern.ch/record/1369546/files/EXO-11-051-pas.pdf.

[24] E. Golowich, J. Hewett, S. Pakvasa and A.A. Petrov, Relating $D^{0}-\bar{D}^{0}$ Mixing and $D 0 \rightarrow \ell^{+} \ell^{-}$with New Physics, Phys. Rev. D 79 (2009) 114030 [arXiv:0903.2830] [INSPIRE].

[25] O. Gedalia, Y. Grossman, Y. Nir and G. Perez, Lessons from Recent Measurements of $D^{0}-\bar{D}^{0}$ Mixing, Phys. Rev. D 80 (2009) 055024 [arXiv:0906.1879] [INSPIRE].

[26] S. Fajfer, Rare decays of D mesons, eConf C 0304052 (2003) WG502 [hep-ph/0306263] [INSPIRE].

[27] S. Fajfer, S. Prelovsek and P. Singer, Rare charm meson decays DtoP $\ell^{+} \ell^{-}$and $c \rightarrow u \ell^{+} \ell^{-}$ in SM and MSSM, Phys. Rev. D 64 (2001) 114009 [hep-ph/0106333] [INSPIRE].

[28] CDF collaboration, F. Margaroli, Measurements of Top Quark Properties at the Tevatron Collider, arXiv:1105.5183 [INSPIRE].

[29] CDF collaboration, K. Potamianos, Top quark physics at CDF, PoS (EPS-HEP2011) 354 [arXiv: 1112.0345].

[30] ATLAS collaboration, J. Carvalho et al., Study of ATLAS sensitivity to FCNC top decays, Eur. Phys. J. C 52 (2007) 999 [arXiv:0712.1127] [INSPIRE].

[31] M. Beneke et al., Top quark physics, hep-ph/0003033 [INSPIRE]. 
[32] A. Deandrea, Atomic parity violation in cesium and implications for new physics, Phys. Lett. B 409 (1997) 277 [hep-ph/9705435] [INSPIRE].

[33] F.J. Botella, G.C. Branco and M. Nebot, Small violations of unitarity, the phase in $B_{s}^{0}-\bar{B}_{s}^{O}$ and visible $t \rightarrow c Z$ decays at the LHC, Phys. Rev. D 79 (2009) 096009 [arXiv:0805.3995] [INSPIRE].

[34] T. Inami and C. Lim, Effects of Superheavy Quarks and Leptons in Low-Energy Weak Processes $K_{L} \rightarrow \mu \bar{\mu}, K^{+} \rightarrow \pi^{+}$Neutrino anti-neutrino and $K^{0} \leftrightarrow \bar{K}^{0}$, Prog. Theor. Phys. 65 (1981) 297 [Erratum ibid. 65 (1981) 1772] [InSPIRE].

[35] G. Cacciapaglia, A. Deandrea and J. Llodra-Perez, $H \rightarrow \gamma \gamma$ beyond the Standard Model, JHEP 06 (2009) 054 [arXiv: 0901.0927] [INSPIRE].

[36] A. Pukhov, CalcHEP 2.3: MSSM, structure functions, event generation, batchs and generation of matrix elements for other packages, hep-ph/0412191 [INSPIRE].

[37] T. Sjöstrand, S. Mrenna and P.Z. Skands, PYTHIA 6.4 Physics and Manual, JHEP 05 (2006) 026 [hep-ph/0603175] [INSPIRE].

[38] M.L. Mangano, M. Moretti, F. Piccinini, R. Pittau and A.D. Polosa, ALPGEN, a generator for hard multiparton processes in hadronic collisions, JHEP 07 (2003) 001 [hep-ph/0206293] [INSPIRE].

[39] F. Maltoni and T. Stelzer, MadEvent: Automatic event generation with MadGraph, JHEP 02 (2003) 027 [hep-ph/0208156] [INSPIRE].

[40] E. Richter-Was et al., ATLFAST 2.0 a fast simulation package for ATLAS, ATL-PHYS-98-131 (1998).

[41] CDF collaboration, Search for Heavy Top $t^{\prime} \rightarrow W q$ in Lepton Plus Jets Events in $\int \mathcal{L} d t=4.6 \mathrm{fb}^{-1}, \mathrm{CDF}$ Conf. Note 10110 presented at Moriond 2010, http://www-cdf.fnal.gov/physics/new/top/confNotes/tprime_CDFnotePub.pdf

[42] CDF collaboration, A. Lister, Search for Heavy Top-like Quarks $t^{\prime} \rightarrow W q$ Using Lepton Plus Jets Events in $1.96 \mathrm{TeV} p \bar{p}$ Collisions, arXiv:0810.3349 [INSPIRE].

[43] A. Atre, G. Azuelos, M. Carena, T. Han, E. Ozcan, et al., Model-Independent Searches for New Quarks at the LHC, JHEP 08 (2011) 080 [arXiv:1102.1987] [INSPIRE]. 